\title{
9. Über die Beugung elektromagnetischer Wellen an parallelen, unendlich langen Kreiszylindern ${ }^{1}$ ); von Franz Záviška.
}

Die Beugung elektromagnetischer Wellen an einem unendlich langen Zylinder von kreiförmigem Querschnitt gehört zu den wenigen Problemen, deren strenge Lösung sich aus den Maxwellschen Gleichungen heraus ohne jede vereinfachende Annahme über die Konstanten des Zylindermateriales durchführen läßt. Die einzige Beschränkung, die man machen muB, betrifft die GröBe des Zylinderradius, der klein gegen die Wellenläge sein soll, was ja bei den Hertzschen Wellen ohnedies erfüllt ist. Das Problem wurde zuerst von Seitz ${ }^{2}$ ) und Ignatowsky ${ }^{3}$ ) gelöst, später hat Schaefer ${ }^{4}$ ) den Fall des dielektrischen Zylinders eingehend diskutiert und sehr interessante Resonanzphänomene gefunden; theoretische Resultate sind von Grossmann ${ }^{5}$ ) experimentell bestätigt worden. Schaefer und Reiche ${ }^{6}$ ) baben auch die Beugung elektromagnetischer Wellen an einem Drahtgitter untersucht, aber unter der Annahme, daB der Abstand der Stäbe so groB gegen die Wellenlänge des einfallenden Lichtes ist, daB man die gegenseitige Beeinflussung derselben vernachlässigen kann, und die Wirkung des ganzen Gitters sich einfach als Summe der Einzelwirkungen der Stäbe ergibt.

1) Teilweise in den "Rozpravy" der böhm. Kaiser-Franz JosefAkademie der Wissenschaften Prag, Nr. 41.1912 abgedruckt.

2) W. Seitz, Ann. d. Phys. 16. p. 746. 1905; 19. p. 554. 1906.

3) W. v. Ignatowsky, Ann. d. Phys. 18. p. 495. 1905.

4) C. Schaefer, Physik. Zeitschr. 10. p. 261. 1909.

5) C. Schaefer u. F. Grossmann, Ann. d. Phys, 31. p. 455. 1910.

6) C. Schaefer n. F. Reiche, Ann. d. Phys. 32. p. 577, 1910; 35. p. 817. 1911 . 
Im folgenden will ich eine exakte Theorie der Beugung elektromagnetischer Wellen an mehreren parallelen Zylindern von kreisförmigem Querschnitt entwickeln, über deren Abstand wir keine Voraussetzung machen, so daB auch die Wirkung der Zylinder aufeinander zur Geltung kommen kann. Freilich werden wir uns hauptsächlich nur mit der Beugung an zwei und drei Zylindern beschäftigen, da, wenn die Zahl der Zylinder wächst, die Rechnung ziemlich unübersichtlich wird. Die einfallende Welle sei eine ebene und geradlinig polarisierte; es genügt zwei Hauptfälle zu untersuchen, nämlich erstens, wenn die elektrische Kraft der einfallenden Welle parallel zu den Zylinderachsen verläuft, zweitens, wenn dieselbe senkrecht zu den letzteren gerichtet ist. Durch Superposition beider so erhaltenen Lösungen ergibt sich sofort die Lösung für den allgemeinen Fall. Die Fortpflanzungsrichtung der einfallenden Welle sei senkrecht zur Ebene der Zylinderachsen. Um die Rechnung zu vereinfachen, wollen wir noch voraussetzen, daB alle Zylinder in jeder Hinsicht identisch sind und gleichen Abstand haben.

Zum SchluB werden wir auch die Beugung an zwei hintereinander stehenden Zylindern untersuchen; die Fortpflanzungsrichtung der einfallenden Welle sei hier parallel zur Ebene der Zylinderachsen. Die Lösung dieses Falles wird uns ermöglichen, einige Abweichungen von den theoretischen Resultaten, die Grossmann bei seinen Messungen der Beugung an einem Zylinder beobachtet hatte, zu erklären.

Wir gehen jetzt an die Betrachtung der einzelnen Fälle.

\section{Die elektrische Kraft parallel zu den Zylinderachsen.}

Wir wählen die Ebene der Zylinderachsen zur $x z$-Ebene eines rechtwinkligen Koordinatensystems, die $z$-Achse sei zu den Zylinderachsen parallel. Anstatt der Koordinaten $x$ und $y$ führen wir ebene Polarkoordinaten $r$ und $\varphi$ ein, und transformieren die Maxwellschen Gleichungen auf die Zylinderkoordinaten $r, \varphi, z$. Da offenbar alle FeldgröBen von $z$ un. abhängig sind und die elektrische Kraft nicht nur in der einfallenden Welle, sondern auch in den reflektierten und in den in die Zylinder eindringenden Wellen parallel zur z-Achse 
verläuft, so nehmen bei Benutzung der üblichen Bezeichnungsweise die Maxwellschen Gleichungen folgende Gestalt an:

$$
\left\{\begin{aligned}
\frac{1}{c}\left(\varepsilon \frac{\partial \mathfrak{E}_{z}}{\partial t}+4 \pi \sigma \mathfrak{E}_{z}\right) & =\frac{1}{r} \frac{\partial}{\partial r}\left(r \mathfrak{E}_{\varphi}\right)-\frac{1}{r} \frac{\partial \mathfrak{Q}_{r}}{\partial \varphi}, \\
\frac{\mu}{c} \frac{\partial \mathfrak{W}_{r}}{\partial t} & =-\frac{1}{r} \frac{\partial \mathbb{E}_{z}}{\partial \varphi}, \\
\frac{\mu}{c} \frac{\partial \mathfrak{F}_{\varphi}}{\partial t} & =\frac{\partial \mathbb{E}_{z}}{\partial r} .
\end{aligned}\right.
$$

Durch Elimination von $\mathfrak{G}_{r}$ und $\mathfrak{E}_{\varphi}$ aus diesen Gleichungen erhalten wir die bekannte Differentialgleichung für $\mathbb{E}_{z}$ :

$$
\frac{\varepsilon \mu}{c^{2}} \frac{\partial^{2} \sqrt{E}}{\partial t^{2}}+\frac{4 \pi \sigma \mu}{c^{2}} \frac{\partial \mathfrak{E}}{\partial t}=\frac{\partial^{2} \mathbb{E}}{\partial r^{2}}+\frac{1}{r} \frac{\partial \mathscr{E}}{\partial r}+\frac{1}{r^{2}} \frac{\partial^{2} \sqrt{E}}{\partial \varphi^{2}},
$$

in welcher der Einfachheit halber $\mathbb{E}$ für $\mathfrak{E}_{z}$ gesetzt wird, und deren Integral unter der Berücksichtigung des Umstandes, daB wir mit rein periodischen Vorgängen zu tun haben, lautet

$$
\mathbb{F}=e^{i \omega t} \sum_{n}^{0, \infty}\left(F_{n}(k r) \cos n \varphi+G_{n}(k r) \sin n \varphi\right),
$$

wo $F_{n}$ und $G_{n}$ allgemeine Besselsche Funktionen $n^{\text {ter }}$ Ordnung und vom Argument $k r$ sind, und $k$ durch die Gleichung

definiert erscheint.

$$
k^{2}=\frac{\mu \varepsilon}{c^{2}} \omega^{2}-\frac{4 \pi \sigma \mu}{c^{2}} \omega i
$$

Nehmen wir Vakuum als AuBenraum und bezeichnen die auf denselben bezüglichen GröBen durch den Index 1, die auf den Innenraum bezüglichen mit 2, so folgt

$$
k_{1}=\frac{2 \pi}{2},
$$

für $k_{2}$ aber bekommt man, wenn die Zylinder aus einem Dielektrikum bestehen,

$$
k_{2}=\frac{2 \pi}{\lambda} \sqrt{\mu_{2} \varepsilon_{2}}
$$

sind dagegen die Zylinder gute Leiter, so daB man $\varepsilon$ gegen $\sigma$ vernachlässigen kann, so folgt

$$
k_{2}=2 \pi \sqrt{\frac{\sigma_{2} \mu_{2}}{c \lambda}}(1-i) \text {. }
$$

Die resultierende elektrische Kraft im AuBenraum besteht aus der elektrischen Kraft der einfallenden Welle und der von

Annalen der Physik. IV. Folge. 40. 
den einzelnen Zylindern ausgehenden Störungswellen. Die Fortpflanzungsrichtung der einfallenden Welle fällt in die $y$-Achse; setzen wir voraus, daB die Welle sich in der Richtung der abnehmenden $y$ bewegt, so folgt

$$
\varepsilon_{1 e}=e^{i\left(\omega t+k_{1} y\right)},
$$

wobei die Amplitude gleich Eins gesetzt wurde. Für die Störungswellen setzen wir den Ausdruck (2); aus der Bedingung, daB derselbe für sehr groBe $r$ eine in der Richtung der steigenden $r$ fortschreitende Welle mit abnehmender Amplitude bezeichnen soll, folgt, wie Ignatowsky zuerst gezeigt hat, daB die Besselschen Funktionen $F_{n}$ und $G_{n}$ sich auf eine einzige reduzieren, die wir mit $Q_{n}$ bezeichnen wollen und die mit den wohlbekannten Besselschen Funktionen erster und zweiter Art, $J_{n}$ und $K_{n}$, mittels der Gleichung

$$
Q_{n}=K_{n}-i-\frac{\pi}{2} J_{n}
$$

zusammenhängt. Sind nun $r_{h}$ und $\varphi_{h}$ Polarkoordinaten des Aufpunktes $M$, bezogen auf den Mittelpunkt $C_{h}$ des $h^{\text {ten }}$ Zylinders

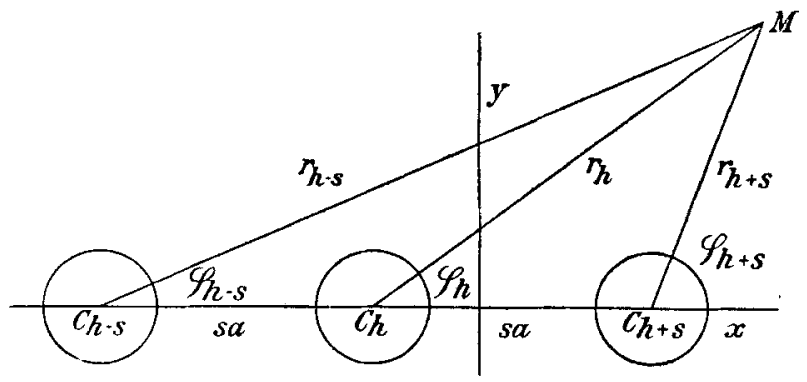

Fig. 1.

(Fig. 1), so bekommt man für die von demselben ausgehende Störungswelle folgenden Ausdruck:

$$
e^{i \omega} \sum_{n}^{0, \infty}\left(A_{h n} \cos n \varphi_{h}+B_{h n} \sin n \varphi_{h}\right) Q_{n}\left(k_{1} r_{h}\right)
$$

und für die resultierende elektrische Kraft im AuBenraum erhält man:

(4) $\mathcal{F}_{1}=e^{i\left(\omega t+k_{1} y\right)}+e^{i \omega t} \sum_{h}^{1, N} \sum_{n}^{0, \infty}\left(A_{h n} \cos n \Psi_{h}+B_{h n} \sin n \varphi_{h}\right) Q_{n}\left(k_{1} r_{h}\right)$, 
wo $N$ die Zahl der Zylinder bedeutet. Die Zahl der Koeffizienten $A_{h n}$ und $B_{h n}$ läBt sich mittels Symmetriebeziehungen noch herabsetzen.

Für innere Punkte kommen nur diejenigen Besselschen Funktionen in Betracht, die für $r_{h}=0$ endlich bleiben; dieser Bedingung genïgt nur die Besselsche Funktion erster Art $J_{n}$, so da $B$ die in den $h^{\text {ten }}$ Zylinder eind ringende Welle durch die Gleichung

$$
\varepsilon_{2 \hbar h}=e^{i \omega t} \sum_{n}^{0, \infty}\left(C_{h n} \cos n \varphi_{h}+D_{h n} \sin n \varphi_{h}\right) J_{n}\left(k_{2} r_{h}\right)
$$

gegeben ist. Auch hier ergeben sich bei Berücksichtigung der Symmetrieverhältnisse Relationen zwischen den Koeffizienten $C_{h n}$ und $D_{h n}$, durch welche die Zahl derselben herabgesetzt wird.

Die übrig bleibenden Koeffizienten $A_{h n}, B_{h n}, C_{h n}$ und $D_{h n}$ sind aus den Grenzbedingungen zu ermitteln. Nach denselben müssen die tangentiellen Komponenten der elektrischen und magnetischen Kraft an der Oberfläche eines jeden Zylinders stetig bleiben. Betrachten wir also die Oberfläche des $h^{\text {ten }}$ Zylinders, so muB für $r_{h}=\varrho$ ( $\rho$ bedeutet den Zylinderradius) und jedes $\varphi_{h}$ gelten:

$$
\left\{\begin{array}{c}
\mathfrak{\mho}_{1}=\mathfrak{E}_{2 h} \\
\left(\mathfrak{S}_{\varphi}\right)_{1}=\left(\mathfrak{S}_{\varphi}\right)_{2 h} ;
\end{array}\right.
$$

statt der letzten Gleichung hat man auch, wie aus der dritten Gleichung (1) folgt,

$$
\frac{\partial \mathbb{E}_{1}}{\partial r_{h}}=\frac{\mu_{1}}{\mu_{2}} \frac{\partial \mathbb{E}_{2 h}}{\partial r_{h}} .
$$

Da die Grenzbedingungen (6) und (6') für ein konstantes $r_{h}$ und beliebiges $\varphi_{h}$ erfüllt sein sollen, so wird es offenbar am zweckmäBigsten sein, den Ausdruck (4) für die resultierende elektrische Kraft im AuBenraum in eine nach dem Kosinus und Sinus des vielfachen $\varphi_{h}$ fortschreitende Fouriersche Reihe, deren Koeffizienten Funktionen von $r_{h}$ wären, zu entwickeln, wie es mit dem Ausdrucke (5) für $\mathcal{F}_{2 h}$ schon der Fall ist. Dies läßt sich verhältnismäBig einfach durchführen. $\mathrm{Zu}$ erst folgt aus einer bekannten Beziehung

$$
\begin{aligned}
e^{i k_{1} y}=e^{i k_{1} r_{h} \sin \varphi_{h}=J_{0}\left(k_{1} r_{h}\right)} & +2 \sum_{n}^{1, \infty} J_{2 n}\left(k_{1} r_{h}\right) \cos 2 n \varphi_{h} \\
& +2 i \sum_{n}^{1, \infty} J_{2 n-1}\left(k_{1} r_{h}\right) \sin (2 n-1) \varphi_{h} .
\end{aligned}
$$


Weiter müssen wir die in Gleichung (4) auftretenden Produkte $Q_{n}\left(k_{1} r_{p}\right) \cos n \varphi_{p}$ und $Q_{n}\left(k_{1} r_{p}\right) \sin n \varphi_{p}$,

wo $p$ eine beliebige ganze und positive, von $h$ verschiedene Zahl, die nicht gröBer als $N$ ist, bedeutet, in der Form einer Fourierschen Reihe darstellen. Ist zuerst $p>h$, so setzen wir $p=h+s$; aus dem $\Delta M C_{h} C_{h+s}$ folgt dann

$$
r_{h+s}^{2}=r_{h}^{2}+s^{2} a^{2}-2 s a r_{h} \cos \varphi_{h},
$$

wo $a$ den gegenseitigen Abstand der Zylinderachsen bezeichnet. Weiter gilt:

$$
\begin{aligned}
& r_{h+s} \cos \varphi_{h+s}=r_{h} \cos \varphi_{h}-s a, \\
& r_{h+s} \sin \varphi_{h+\varepsilon}=r_{h} \sin \varphi_{h} .
\end{aligned}
$$

Addiert man die letzte mit $i$ multiplizierte Gleichung zur vorstehenden, so folgt:

ähnlich folgt:

$$
r_{h+s} e^{i \varphi_{h+s}}=r_{h} e^{i \varphi_{h}}-s a,
$$

$$
r_{h+s} e^{-i \varphi_{h+s}}=r_{h} e^{-i \varphi_{h}}-s a,
$$

und aus beiden letzten Gleichungen ergibt sich, wenn $r_{h}<s a$ ist:

$$
e^{i \varphi_{h+s}}=-\left(\frac{s a-r_{h} e^{i \varphi_{h}}}{s a-r_{h} e^{-i \varphi_{h}}}\right)^{1 / 2}
$$

das Zeichen der rechten Seite wird dadurch bestimmt, daB für $\varphi_{h}=0$ die Wurzel gleich -1 sein mub; es ist dann nämlich $\varphi_{h+s}=\pi$. Daraus folgt

$$
\begin{aligned}
& Q_{n}\left(k_{1} r_{h+s}\right) e^{-i n \varphi_{h+s}} \\
& =(-1)^{n}\left(\frac{s a-r_{h} e^{i \varphi_{h}}}{s a-r_{h} e^{-i \varphi_{h}}}\right)^{-\frac{n}{2}} Q_{n}\left(k_{1} \sqrt{r_{h}^{2}+s^{2} a^{2}-2 s a r_{h} \cos \varphi_{h}}\right) .
\end{aligned}
$$

Nun läBt sich die rechte Seite in eine Neumannsche Reihe entwickeln. Nach einer Formel, die Graf ${ }^{1}$ ) zuerst angegeben hat, hat man

$$
\left\{\begin{array}{c}
\left(\frac{s a-r_{h} e^{i \varphi_{h}}}{s a-r_{h} e^{-i \varphi_{h}}}\right)^{-\frac{n}{2}} F_{n}\left(k_{1} \sqrt{r_{h}{ }^{2}+s^{2} a^{2}-2 s a r_{h} \cos \varphi_{h}}\right) \\
=\sum_{m}^{-\infty, \infty} F_{m+n}\left(k_{1} s a\right) J_{m}\left(k_{1} r_{h}\right) e^{i m \varphi_{h}}
\end{array}\right.
$$

1) J. H. Graf, Mathemat. Annalen 43. p. 136. 1893. 
wo $F_{n}$ eine beliebige Besselsche Funktion sein kann, von der wir hier voraussetzen wollen, daB sie für ein reelles Argument auch reell ist. Die rechts stehende Reihe ist sicher konvergent, sobald $r_{h}<s a$ ist, was in der Nähe der Oberfläche des $h^{\text {ten }}$ Zylinders immer gilt. Diejenigen Besselschen Funktionen, deren Ordnung durch eine negative Zahl gegeben ist, sind durch die Gleichung

$$
F_{-n}=(-1)^{n} F_{n}
$$

definiert. Durch Trennung der reellen Bestandteile von den imaginären erbält man leicht die Entwickelungen für

$$
F_{n}\left(k_{1} r_{h+s}\right) \cos n \varphi_{h+s} \text { und } F_{n}\left(k_{1} r_{h+s}\right) \sin n \varphi_{h+s} \text {. }
$$

Setzt man nun zuerst $F_{n}=K_{n}$, dann $F_{n}=J_{n}$, so folgen nach einer einfachen Rechnung auch die Entwickelungen für

$$
Q_{n}\left(k_{1} r_{h+s}\right) \cos n \varphi_{h+s} \quad \text { und } Q_{n}\left(k_{1} r_{h+s}\right) \sin n \varphi_{h+s} \text {. }
$$

Wir schreiben

$$
\left\{\begin{array}{l}
Q_{n}\left(k_{1} r_{h+s}\right) \cos n \varphi_{h+s}=(-1)^{n} \sum_{m}^{0, \infty} a_{n m}^{(s)} J_{m}\left(k_{1} r_{h}\right) \cos m \varphi_{h} \\
Q_{n}\left(k_{1} r_{h+s}\right) \sin n \varphi_{h+s}=(-1)^{n-1} \sum_{m}^{1, \infty} b_{n m}^{(s)} J_{m}\left(k_{1} r_{h}\right) \sin m \varphi_{h}
\end{array}\right.
$$

wo

$$
\left\{\begin{array}{l}
a_{n 0}^{(s)}=Q_{n}\left(s k_{1} a\right) \\
a_{n m}^{(s)}=Q_{n+m}\left(s k_{1} a\right)+(-1)^{m} Q_{n-m}\left(s k_{1} a\right) \\
b_{n m}^{(s)}=Q_{n+m}\left(s k_{1} a\right)+(-1)^{m-1} Q_{n-m}\left(s k_{1} a\right) .
\end{array}\right.
$$

Durch Derivation der Gleichungen (8) erhält man weitere Entwickelungen in der Form:

$$
\left\{\begin{aligned}
& \frac{1}{k_{1}} \frac{\partial}{\partial r_{h}}\left(Q_{n}\left(k_{1} r_{h+s}\right) \cos n \varphi_{h+s}\right) \\
&=(-1)^{n} \sum_{m}^{0, \infty} a_{n m}^{(s)} J_{m}^{\prime}\left(k_{1} r_{h}\right) \cos m \varphi_{h} \\
& \frac{1}{k_{1}} \frac{\partial}{\partial r_{h}}\left(Q_{n}\left(k_{1} r_{h+s}\right) \sin n \varphi_{h+s}\right) \\
&=(-1)^{n-1} \sum_{m}^{1+\infty} b_{n m}^{(s)} J_{m}^{\prime}\left(k_{1} r_{h}\right) \sin m \varphi_{h}
\end{aligned}\right.
$$

wo

gesetzt wurde.

$$
J_{m}^{\prime}(x)=\frac{d J_{m}(x)}{d x}
$$


Ist nun $p<h$, also $p=h-s$, so hat man zuerst:

$$
e^{i \varphi_{h-s}}=\left(\frac{s a+r_{h} e^{-i \varphi_{h}}}{s a+r_{h} e^{i \varphi_{h}}}\right)^{-1 / 2},
$$

solange $r_{h}<s a$ ist. Setzt man nun

$$
\varphi_{h}=\pi-\vartheta,
$$

so findet man in derselben Weise wie früher folgende Entwickelungen:

$$
\left\{\begin{array}{l}
Q_{n}\left(k_{1} r_{h-s}\right) \cos n \varphi_{h-s}=\sum_{m}^{0, \infty}(-1)^{m} \quad a_{n m}^{(s)} J_{m}\left(k_{1} r_{h}\right) \cos m \varphi_{h}, \\
Q_{n}\left(k_{1} r_{h-s}\right) \cos n \varphi_{h-s}=\sum_{m}^{1, \infty}(-1)^{m-1} b_{m m}^{(s)} J_{m}\left(k_{1} r_{h}\right) \sin m \varphi_{h} .
\end{array}\right.
$$

Führt man die Entwickelungen (8) und (10) in die Gleichung (4) ein, so bekommt man leicht aus den Grenzbedingungen (6) und $\left(6^{\prime}\right)$ lineare Beziehungen zwischen den Koeffizienten $A_{h n}$ usw., die zur Bestimmung derselben dienen können.

Um uns aber nicht in Allgemeinheiten zu verlieren, wollen wir zur Betrachtung spezieller Fälle übergehen. Setzen wir

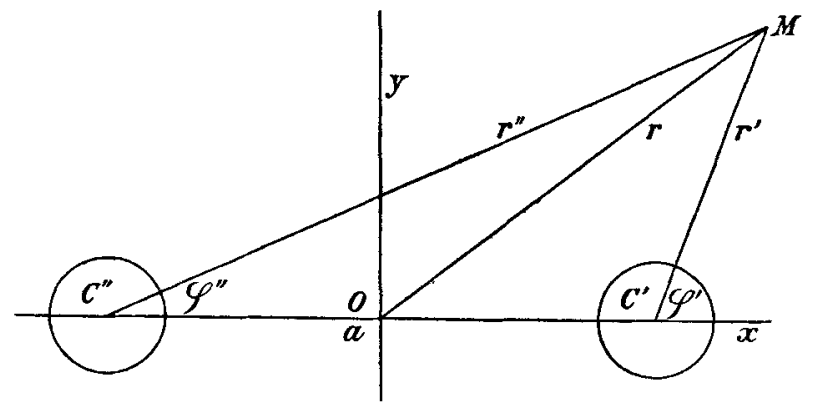

Fig. 2.

voraus, dab die Beugung an zwei parallelen Zylindern vor sich geht. Es seien $r^{\prime}$ und $\varphi^{\prime}$ die Polarkoordinaten des Aufpunktes $M$ in bezug auf den Mittelpunkt $C^{\prime}$ des rechten Zylinders (Fig. 2), $r^{\prime \prime}, \varphi^{\prime \prime}$ in bezug auf den Mittelpunkt $C^{\prime \prime}$ des linken Zylinders, so erhält man für die resultierende elektrische 
Kraft im AuBenraum $\mathfrak{F}_{1}$ aus der Gleichung (4) folgenden Ausdruck:

$$
\begin{aligned}
\tilde{F}_{1}=e^{i\left(\omega t+k_{1} y\right)} & +e^{i \omega t} \sum_{n}^{0 \cdot \infty}\left(A_{n}^{\prime} \cos n \varphi^{\prime}+B_{n}^{\prime} \sin n \varphi^{\prime}\right) Q_{n}\left(k_{1} r^{\prime}\right) \\
& +e^{i \omega t} \sum_{n}^{0, \infty}\left(A_{n}^{\prime \prime} \cos n \varphi^{\prime \prime}+B_{n}^{\prime \prime} \sin n \varphi^{\prime \prime}\right) Q_{n}\left(k_{1} r^{\prime \prime}\right) .
\end{aligned}
$$

Da nun offenbar die $y z$-Ebene eine Symmetrieebene für die elektrische Kraft ist, so $\mathrm{muB}$ gelten:

$$
A_{n}{ }^{\prime}=(-1)^{n} A_{n}{ }^{\prime \prime}=A_{n}, \quad B_{n}{ }^{\prime}=(-1)^{n-1} B_{n}{ }^{\prime \prime}=B_{n},
$$

es ist also:

$$
\text { (11) }\left\{\begin{array}{l}
\mathbb{E}_{1}=e^{i\left(\omega t+k_{1} y\right)} \\
+e^{i \omega t} \sum_{n}^{0, \infty} A_{n}\left(Q_{n}\left(k_{1} r^{\prime}\right) \cos n \varphi^{\prime}+(-1)^{n} \quad Q_{n}\left(k_{1} r^{\prime \prime}\right) \cos n \varphi^{\prime \prime}\right) \\
+e^{i \omega t} \sum_{n}^{1, \infty} B_{n}\left(Q_{n}\left(k_{1} r^{\prime}\right) \sin n \varphi^{\prime}+(-1)^{n-1} Q_{n}\left(k_{1} r^{\prime \prime}\right) \sin n \varphi^{\prime \prime}\right)
\end{array}\right.
$$

Für die in den rechten Zylinder eindringende Welle setzen wir nach Gleichung (5)

$$
\xi_{2}^{\prime}=e^{i \omega t} \sum_{n}^{0, \infty}\left(C_{n} \cos n \varphi^{\prime}+D_{n} \sin n \varphi^{\prime}\right) J_{n}\left(k_{2} r^{\prime}\right),
$$

und daraus folgt für die in den linken Zylinder eindringende Welle der Ausdruck:

$$
\mathbb{F}_{2}^{\prime \prime}=e^{i \omega t} \sum_{n}^{0, \infty}(-1)^{n}\left(C_{n} \cos n \varphi^{\prime \prime}-D_{n} \sin n \varphi^{\prime \prime}\right) J_{n}\left(k_{2} r^{\prime \prime}\right),
$$

wobei die Symmetrieverhältnisse schon berücksichtigt sind.

Die Grenzbedingungen (6) und $\left(6^{\prime}\right)$ sollen nun für die Oberfläche beider Zylinder erfüllt werden. Man sieht aber, daß es wegen der Symmetrie der Ausdrücke (11), (12) und (12') genügt, dieselben nur für die Oberfläche des einen von ihnen, z. B. des rechten, aufzustellen. Aus den Gleichungen (10) folgt dann:

$$
\left\{\begin{array}{l}
Q_{n}\left(k_{1} r^{\prime \prime}\right) \cos n \varphi^{\prime \prime}=\sum_{n}^{0}(-1)^{m} a_{n m} J_{m}\left(k_{1} r^{\prime}\right) \cos m \varphi^{\prime} \\
Q_{n}\left(k_{1} r^{\prime \prime}\right) \sin n \varphi^{\prime \prime}=\sum_{m}^{1, \infty}(-1)^{m-1} b_{n m} J_{m}\left(k_{1} r^{\prime}\right) \sin m \varphi^{\prime}
\end{array}\right.
$$


wo

$$
\left\{\begin{array}{l}
a_{n 0}=Q_{n}\left(k_{1} a\right), \\
a_{n m}=Q_{n+m}\left(k_{1} a\right)+(-1)^{m} Q_{n-m}\left(k_{1} a\right), \\
b_{n m}=Q_{n+m}\left(k_{1} a\right)+(-1)^{m-1} Q_{n-m}\left(k_{1} a\right)
\end{array}\right.
$$

gesetzt wurde.

Die linearen Gleichungen für $A_{n}, B_{n}$ usw., die sich aus den Grenzbedingungen (6) und $\left(6^{\prime}\right)$ ergeben, zerfallen in zwei voneinander unabhängige Systeme. Setzt man der Kürze halber

$$
\left\{\begin{array}{l}
k_{1} \varrho=p_{1}, \quad k_{2} \varrho=p_{2}, \\
\frac{\mu_{1}}{\mu_{2}} \frac{k_{2}}{k_{1}}=\frac{\mu_{1}}{\mu_{2}} \frac{p_{2}}{p_{1}}=\eta,
\end{array}\right.
$$

so lautet das erste System

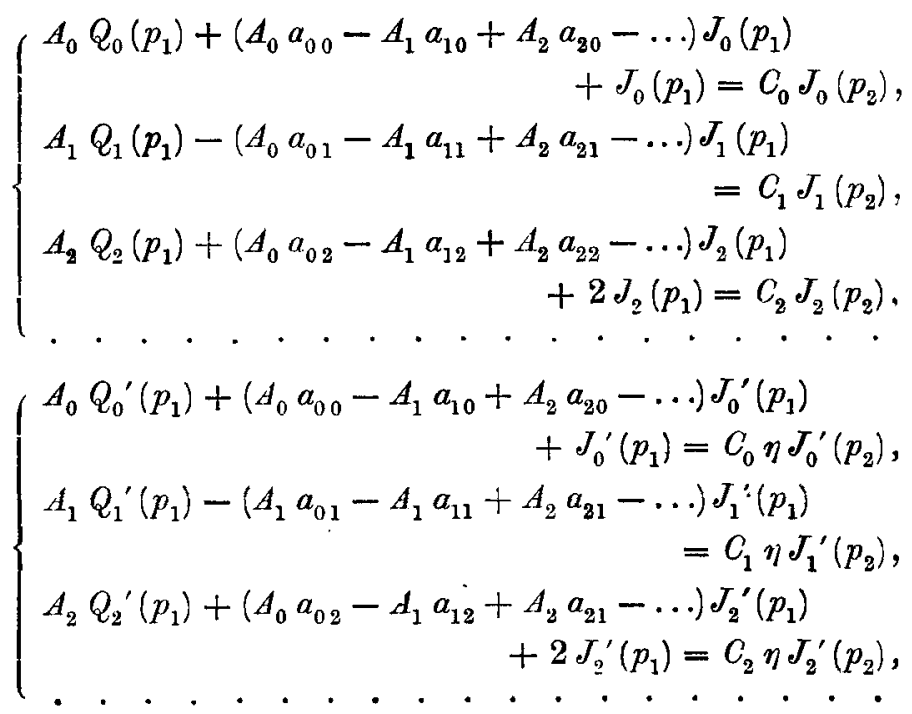

und ähnlich erhält man für das zweite System:

$$
\left\{\begin{aligned}
& B_{1} Q_{1}\left(p_{1}\right)+\left(B_{1} b_{11}-B_{2} b_{21}\right.+\ldots) J_{1}\left(p_{1}\right) \\
&+2 i J_{1}\left(p_{1}\right)=D_{1} J_{1}\left(p_{2}\right) \\
& B_{2} Q_{2}\left(p_{1}\right)-\left(B_{1} b_{12}-B_{2} b_{22}+\ldots\right) J_{2}\left(p_{1}\right)=D_{2} J_{2}\left(p_{2}\right) \\
& B_{3} Q_{3}\left(p_{1}\right)+\left(B_{1} b_{13}-B_{2} b_{23}+\ldots\right) J_{3}\left(p_{1}\right) \\
&+2 i J_{3}\left(p_{1}\right)=D_{3} J_{3}\left(p_{2}\right)
\end{aligned}\right.
$$




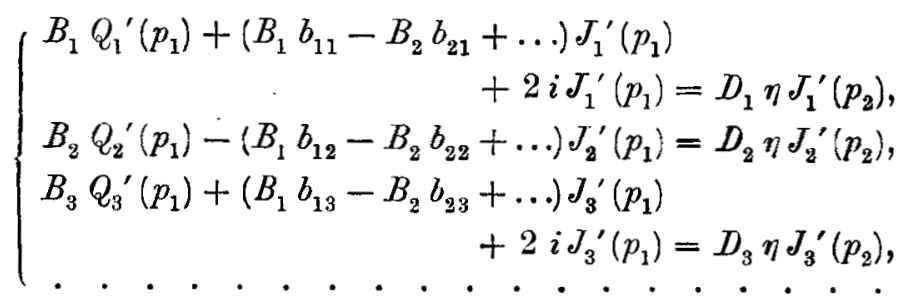

In erster Reihe handelt es sich um dis Bestimmung der Koeffizienten $A_{n}$ und $B_{n}$, welche im Ausdrucke (11) für die elektrische Kraft im Außenraum auftreten. Eliminiert man also $C_{n}$ und $D_{n}$ aus den letzten Gleichungen und setzt man der Kürze halber

so folgt

$$
\left\{\begin{array}{l}
J_{n}^{\prime}\left(p_{1}\right) J_{n}\left(p_{2}\right)-\eta J_{n}\left(p_{1}\right) J_{n}^{\prime}\left(p_{2}\right)=G_{n}, \\
Q_{n}^{\prime}\left(p_{1}\right) J_{n}\left(p_{2}\right)-\eta Q_{n}\left(p_{1}\right) J_{n}^{\prime}\left(p_{2}\right)=H_{n},
\end{array}\right.
$$

$$
\begin{aligned}
& \left\{\begin{array}{l}
A_{0} H_{0}+\left(A_{0} a_{00}-A_{1} a_{10}+A_{2} a_{20}-\ldots\right) G_{0}+G_{0}=0 \\
A_{1} H_{1}-\left(A_{0} a_{01}-A_{1} a_{11}+A_{2} a_{21}-\ldots\right) G_{1}=0 \\
A_{2} H_{2}+\left(A_{0} a_{02}-A_{1} a_{12}+A_{2} a_{22}-\ldots\right) G_{2}+2 G_{2}=0 \\
. . . . . . . . . . . . .
\end{array}\right.
\end{aligned}
$$

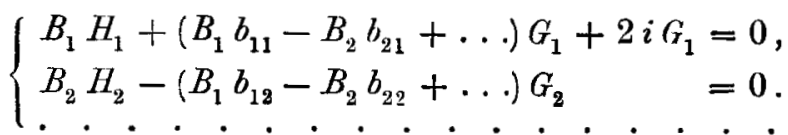

Die Auflösung dieser Gleichungen gestaltet sich sehr einfach, wenn das Verhältnis $\varrho / \lambda$, und also auch $p_{1}$, hinreichend klein ist, weil dann die Koeffizienten $A_{n}$ und $B_{n}$ mit steigendem Index $n$ rasch abnehmen. Beschränkt man sich nämlich in den bekannten Reihen für die Zylinderfunktionen $K_{n}\left(p_{1}\right)$ und $J_{n}\left(p_{1}\right)$ stets auf das erste Glied, was für kleine $p_{1}$ als erste Annäherung zu betrachten ist, so folgt aus den Gleichungen (18) für $n>1$

$$
\begin{aligned}
& G_{n}=\frac{1}{n !} \frac{p_{1}^{n-1}}{2^{n}}\left(n J_{n}\left(p_{2}\right)-\frac{p_{z}}{\mu_{9}} J_{n}^{\prime}\left(p_{2}\right)\right), \\
& H_{n}=-(n-1) ! \frac{2^{n-1}}{p_{1}^{n+1}}\left(n J_{n}\left(p_{2}\right)+\frac{p_{9}}{\mu_{2}} J_{n}^{\prime}\left(p_{2}\right)\right)
\end{aligned}
$$

Man sieht also, daB die Koeffizienten $A_{n}$ und $B_{n}$ im all-

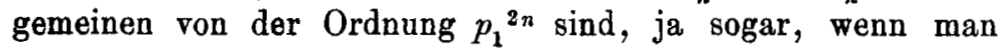


auch $p_{3}$ als klein betrachten darf, und $\mu_{2}$ gleich eins ist, was bei dielektrischen Zylindern und bei äuBerst feinen, unmagnetischen Metalldrähten erfüllt ist, erhält man in der hier benutzten Annäherung $G_{n}=0$, so daB die Werte von $A_{n}$ und $B_{n}$ noch kleiner ausfallen.

Daraus folgt nun eine einfache Methode zur Auflösung der Gleichungen (19) und $\left(19^{\prime}\right)$. Vernachlässigt man in der ersten Gleichung (19) alle Glieder mit $A_{1}, A_{2}, \ldots$, so erhält man durch die Auflösung derselben den ersten Näherungswert für $A_{0}$. Setzt man diesen Wert in die zweite Gleichung (19), in der man nur die Glieder mit $A_{1}$ behält, so folgt daraus der erste Näherungswert für $A_{1}$, in gleicher Weise bestimmt man den ersten Näherungswert für $A_{2}$ usw. Wenn man dann die so erhaltenen Werte für $A_{1}, A_{2}, \ldots$ in die erste Gleichung (19) einführt, so bekommt man den zweiten Näherungswert für $A_{0}$, ähnlich folgt aus der zweiten Gleichung (19) der zweite Näherungswert für $A_{1}$ usw. Das Verfahren führt rasch zum Ziele; manchmal genügt es schon, nur die ersten Näherungswerte zu kennen.

Das Feld im AuBenraum ist durch den Ausdruck (11) gegeben, von dem nur der reelle Teil zu berücksichtigen ist. Setzt man also

$$
\begin{aligned}
& A_{n}=\alpha_{n}+i \gamma_{n}, \\
& B_{n}=\beta_{n}+i \delta_{n},
\end{aligned}
$$

so bringt man $\xi_{1}$ leicht auf die Form

$$
E_{1}=P \cos \omega t-Q \sin \omega t
$$

wo zur Abkürzung gesetzt ist:

$$
\left\{\begin{aligned}
P=\cos k_{1} y & +\sum K_{n}\left(k_{1} r^{\prime}\right)\left(\alpha_{n} \cos n \varphi^{\prime}+\beta_{n} \sin n \varphi^{\prime}\right) \\
& +\frac{\pi}{2} \sum J_{n}\left(k_{1} r^{\prime}\right)\left(\gamma_{n} \cos n \varphi^{\prime}+\delta_{n} \sin n \varphi^{\prime}\right) \\
& +\sum(-1)^{n} K_{n}\left(k_{1} r^{\prime \prime}\right)\left(\alpha_{n} \cos n \varphi^{\prime \prime}-\beta_{n} \sin n \varphi^{\prime \prime}\right) \\
& +\frac{\pi}{2} \sum(-1)^{n} J_{n}\left(k_{1} r^{\prime \prime}\right)\left(\gamma_{n} \cos n \varphi^{\prime \prime}-\delta_{n} \sin n \varphi^{\prime \prime}\right) \\
Q=\sin k_{1} y & +\sum K_{n}\left(k_{1} r^{\prime}\right)\left(\gamma_{n} \cos n \varphi^{\prime}+\delta_{n} \sin n \varphi^{\prime}\right) \\
& -\frac{\pi}{2} \sum J_{n}\left(k_{1} r^{\prime}\right)\left(\alpha_{n} \cos n \varphi^{\prime}+\beta_{n} \sin n \varphi^{\prime}\right) \\
& +\sum(-1)^{n} K_{n}\left(k_{1} r^{\prime \prime}\right)\left(\gamma_{n} \cos n \varphi^{\prime \prime}-\delta_{n} \sin n \varphi^{\prime \prime}\right) \\
& -\frac{\pi}{2} \sum(-1)^{n} J_{n}\left(k_{1} r^{\prime \prime}\right)\left(\alpha_{n} \cos n \varphi^{\prime \prime}-\beta_{n} \sin n \varphi^{\prime \prime}\right)
\end{aligned}\right.
$$


Bei experimentellen Messungen kommt es hauptsächlich auf den zeitlichen Mittelwert von $\mathfrak{F}_{1}^{2}, d$. h. auf

$$
{\overline{F_{1}}}_{1}^{2}=\frac{1}{t} \int_{0}^{\tau}{\mathfrak{F}_{1}}^{2} d t
$$

an; $\tau$ bezeichnet die Schwingungsperiode. Nach der Gleichung (20) ist einfach

$$
{\overline{\aleph_{1}}}_{1}^{2}=\frac{1}{2}\left(P^{2}+Q^{2}\right) \text {. }
$$

Der Ausdruck für $\overline{\mathbb{E}}_{1}^{2}$, zu dem man nach Einführung von $P$ und $Q$ aus den Gleichungen (20') gelangt, ist für die Diskussion nicht geeignet. Man kann ihm eine einfachere Gestalt geben, indem man voraussetzt, daB die Entfernungen $r^{\prime}$ und $r^{\prime \prime}$ des Aufpunktes von beiden Zylinderachsen hinreichend groB gegen die Wellenlänge sind, so daB man für die Besselschen Funktionen $J_{n}$ und $K_{n}$ die bekannten asymptotischen Darstellungen benutzen darf. Man findet dann:

$$
\left\{\begin{array}{r}
P=\cos k_{1} y-\frac{1}{2} \sqrt{\frac{\lambda}{r^{\prime}}}\left(S^{\prime} \sin \psi^{\prime}-T^{\prime} \cos \psi^{\prime}\right) \\
-\frac{1}{2} \sqrt{\frac{\lambda}{r^{\prime \prime}}}\left(S^{\prime \prime} \sin \psi^{\prime \prime}-T^{\prime \prime} \cos \psi^{\prime \prime}\right), \\
Q=\sin k_{1} y-\frac{1}{2} \sqrt{\frac{\lambda}{r^{\prime}}}\left(S^{\prime} \cos \psi^{\prime}+T^{\prime} \sin \psi^{\prime}\right) \\
-\frac{1}{2} \sqrt{\frac{\lambda}{r^{\prime \prime}}}\left(S^{\prime \prime} \cos \psi^{\prime \prime}+T^{\prime \prime} \sin \psi^{\prime \prime}\right),
\end{array}\right.
$$

wo gesetzt wurde:

$$
\begin{gathered}
\psi^{\prime}=k_{1} r^{\prime}-\frac{\pi}{4}, \quad \psi^{\prime \prime}=k_{1} r^{\prime \prime}-\frac{\pi}{4} \\
S^{\prime}=\sum_{m}^{0, \infty}(-1)^{m}\left(\alpha_{2 m} \cos 2 m \varphi^{\prime}+\beta_{2 m} \sin 2 m \varphi^{\prime}\right. \\
\left.-\gamma_{2 m+1} \cos (2 m+1) \varphi^{\prime}-\delta_{2 m+1} \sin (2 m+1) \varphi^{\prime}\right) \\
T^{\prime}=\sum_{m}^{0, \infty}(-1)^{m}\left(\gamma_{2 m} \cos 2 m \varphi^{\prime}+\delta_{2 m} \sin 2 m \varphi^{\prime}\right. \\
\left.+\alpha_{2 m+1} \cos (2 m+1) \varphi^{\prime}+\beta_{2 m+1} \sin (2 m+1) \varphi^{\prime}\right)
\end{gathered}
$$

Die Ausdrücke für $S^{\prime \prime}$ und $T^{\prime \prime}$ folgen aus denen für $S^{\prime}$ und $T^{\prime}$, wenn man in dieselben $180^{\circ}-\varphi^{\prime \prime}$ statt $\varphi^{\prime}$ einführt. 
Aus der Gleichung (21) ergibt sich

$$
\left\{\begin{array}{c}
{\overline{\mathbb{E}_{1}}}^{2}=\frac{1}{2}+\frac{\lambda}{4 \sqrt{r^{\prime} r^{\prime \prime}}}\left(\left(S^{\prime} S^{\prime \prime}+T^{\prime} T^{\prime \prime}\right) \cos \left(\psi^{\prime}-\psi^{\prime \prime}\right)\right. \\
\left.-\left(S^{\prime} T^{\prime \prime}-S^{\prime \prime} T^{\prime}\right) \sin \left(\psi^{\prime}-\psi^{\prime \prime}\right)\right) \\
+\frac{\lambda}{8 r^{\prime}}\left(S^{\prime 2}+T^{\prime 2}\right)+\frac{\lambda}{8 r^{\prime \prime}}\left(S^{\prime \prime 2}+T^{\prime \prime 2}\right) \\
-\frac{1}{2} \sqrt{\frac{\lambda}{r^{\prime}}}\left(S^{\prime} \sin \left(k_{1} y+\psi^{\prime}\right)-T^{\prime} \cos \left(k_{1} y+\psi^{\prime}\right)\right) \\
-\frac{1}{2} \sqrt{\frac{\lambda}{r^{\prime \prime}}}\left(S^{\prime \prime} \sin \left(k_{1} y+\psi^{\prime \prime}\right)-T^{\prime \prime} \cos \left(k_{1} y+\psi^{\prime \prime}\right)\right)
\end{array}\right.
$$

Diese Gleichung besagt, daB sich die mittlere Feldintensität mit der Lage des Aufpunktes periodisch ändert, oder daB sich Interferenzstreifen bilden. Aus der Form derselben sieht man, $\mathrm{daB}$ es am einfachsten ist, den Verlauf der Feldintensität an denjenigen Kurven zu betrachten, an welchen die Differenz $\psi^{\prime}-\psi^{\prime \prime}$ oder auch $r^{\prime}-r^{\prime \prime}$ konstant bleibt. Es sind Hyperbeln (im Raume hyperbolische Zylinder), die in Gerade, welche durch den Mittelpunkt verlaufen, übergehen, sobald die Entfernung des Aufpunktes von den beiden Zylinderachsen gegen ihren $A b s t a n d$ groB ist. Unter derselben Bedingung bleiben auch die Werte von $S^{\prime}, T^{\prime \prime}, S^{\prime \prime}, T^{\prime \prime}$ nahezu konstant, wenn sich der Aufpunkt auf einer solchen Geraden bewegt, denn die Winkel $\varphi^{\prime}$ und $\varphi^{\prime \prime}$ ändern sich langsam, und auBerdem nehmen die Koeffizienten $\alpha_{m}, \beta_{n n}$ usw. mit steigendem Index $m$ rasch ab. Wächst nun beim Übergange von einem Maximum oder Minimum der Feldintensität zum nächstfolgenden $y$ um $\Delta y$, $\psi^{\prime}$ (oder $\psi^{\prime \prime}$ ) um $\Delta \psi^{\prime}$, so folgt aus Gleichung (23) mit groBer Annäherung

$$
k_{1} \Delta y+\Delta \psi^{\prime}=2 \pi .
$$

Es sei nun $r$ die Entfernung des Aufpunktes $M$ rom Mittelpunkte $0, \chi$ der Winkel, den die Verbindungslinie beider dieser Punkte mit der negativen $y$-Achse einschlieBt - wir wollen $\chi$ den „Beugungswinkel" nennen -; man hat dann $y=-r \cos \chi$, weiter folgt aus der ersten Gloichung (22')

$$
\Delta \psi^{\prime}=k_{1} \Delta r^{\prime},
$$

oder auch, weil $r^{\prime}$ grob gegen den Abstand beider Zylinder ist,

$$
\Delta \psi^{\prime}=k_{\mathrm{I}} \Delta r \text {. }
$$


So bekommt man aus der Gleichung (24) für den Abstand $\Delta r$ zweier aufeinanderfolgender Maxima oder Minima folgenden Wert:

$$
\Delta r=\frac{\lambda}{1}-\frac{\cos \chi}{\cos }
$$

Die Interferenzstreifen liegen also am häufigsten nebeneinander, wenn $\chi=\pi$ ist, d. h. in der Symmetrieebene, vor den Zylindern; es ist dann $\Delta r=\lambda / 2$. In der Ebene beider Zylinderachsen ist $\chi=\pi / 2$, also $\Delta r=\lambda$, endlich in der Symmetrieebene, aber hinter den Zylindern, ist $\chi=0$, und $\Delta r$ ist unendlich groB, hier also kommen keine Interferenzstreifen zustande, wenigstens nicht für Entfernungen, die groß gegen $a$ und $\lambda$ sind.

Ganz denselben Beziehungen begegnet man auch bei der Untersuchung der Beugung elektromagnetischer Wellen an einem einzigen Zylinder; auch da ist der Abstand zweier aufeinanderfolgender Maxima oder Minima bei festem $\chi$ durch die Gleichung (24') gegeben. Überhaupt sieht man aus dem allgemein gültigen Ausdrucke (4) für die resultierende elektrische Kraft im AuBenraum, daß man wieder zur Gleichung (24) gelangt, sobald die Entfernungen des Aufpunktes von den Zylindern gegen die Wellenlänge und gegen den $\mathrm{Ab}$. stand der beiden äuBeren Zylinder groB sind. Speziell folgt auch für zwei Zylinder, daB, wenn der Abstand der Zylinder klein ist, das Feld im AuBenraum im groBen und ganzen demjenigen eines einzigen Zylinders ähnlich verläuft, weil die Phasendifferenz der beiden von den Zylindern ausgehenden Störungswellen klein ist. Frst mit wachsendem Abstand der Zylinder tritt der EinfluB der Interferenz beider Wellen zutage; so z. B. bilden sich in der Symmetrieebene, hinter den Zylindern, Interferenzstreifen, deren Zahl aber endlich bleibt und mit zunehmendem Abstand der Zylinder auch zunimmt.

Um nun die Wirkung beider Zylinder aufeinander zu verfolgen, wollen wir voraussetzen, $\mathrm{daB}$ beide Zylinder dünn sind, daB also $p_{1}$ hinreichend klein ist. Die Rechnung wird dadurch bedeutend vereinfacht, denn es fallen dann die Koeffizienten $A_{n}$ und $B_{n}$, wie wir schon früher gezeigt haben, sehr rasch ab, so dab man in erster Annäherung alle gegen $A_{0}$ vernachlässigen darf, wenigstens, insofern es sich um die Be- 
stimmung der Feldintensität in Punkten, die nicht allzu nahe an der Oberfläche der Zylinder liegen, handelt. Es folgt dann aus der ersten Gleichung (19)

$$
A_{0}=-\frac{G_{0}}{H_{0}+Q_{0}\left(k_{1} a\right) \theta_{0}},
$$

wo die Werte von $G_{0}$ und $H_{0}$ durch die Gleichungen (18) gegeben sind, in welche $n=0$ zu setzen ist.

Für die weitere Diskussion müssen wir noch auseinanderhalten, ob die Beugung an dielektrischen oder an leitenden Zylindern erfolgt. Im ersten Falle ist $p_{2}=p_{1} \sqrt{\varepsilon}$, es ist also auch klein; weiter ist nach der letzten Gleichung (15) $\eta=\sqrt{\varepsilon \text {, }}$ wenn man der Einfachheit wegen $\mu_{2}=1$ setzt, woraus folgt

$$
H_{0}=-\frac{1}{p_{1}}, \quad G_{0}=\frac{p_{1}}{2}(\varepsilon-1),
$$

und mit derselben Genauigkeit folgt weiter

$$
A_{0}=\frac{\varepsilon-1}{2} p_{1}{ }^{2}\left(1+\frac{\varepsilon-1}{2} p_{1}{ }^{2} Q_{0}\left(k_{1} a\right)\right)
$$

Das Glied mit $Q_{0}\left(k_{1} a\right)$ entspricht der gegenseitigen Beeinflussung beider Zylinder. Bedenkt man, daB $Q_{0}\left(k_{1} a\right)$ für kleine Werte von $a$ von der Ordnung $\log k_{1} a$ ist, und daB $k_{1} a$ nimmer kleiner sein kann als $2 p_{1}$, so sieht man, daB auch bei kleiner Entfernung beider Zylinder die Wirkung derselben aufeinander gering ist, und daB man das Feld im AuBenraum mit großer Annäherung einfach als durch Übereinanderlagerung beider Felder, die die Zylinder für sich allein hervorrufen würden, entstanden denken kann. Aus der letzten Gleichung folgt auch, daB sich die gegenseitige Beeinflussung beider Zylinder bei wachsendem Abstand a periodisch - natürlich mit sinkender Amplitude - ändert.

Ganz anders stehen die Verhältnisse, wenn beide Zylinder gute Leiter sind. Wegen des groben Wertes der Leitungs. fähigkeit, die in elektrostatischen Einheiten ausgedrückt wird, ist $k_{2}$, und also auch $\eta$, sehr groB, so daB man, den Fall äuBerst feiner Metalldrähte ausgenommen, in den Ausdrücken (18) für $G_{0}$ und $H_{0}$ die mit $\eta$ nicht behafteten Glieder ohne Bedenken streichen kann. Man erhält dann:

$$
A_{0}=-\frac{J_{0}\left(p_{1}\right)}{Q_{0}} \frac{\left(p_{1}\right)+Q_{0}\left(k_{1} a\right)}{,}
$$


eine Formel, aus welcher alle den Zylindern zugehörigen Konstanten verschwunden sind, und welche für unendlich gute Leiter streng gültig wäre. Aus dieser Gleichung sieht man auch, $\mathrm{daB}$ die gegenseitige Beeinflussung der beiden $Z$ ylinder in diesem Falle recht beträchtlich sein kann, denn für kleine Werte von $a$ sind beide im Nenner auftretenden Glieder von derselben Ordnung; erst für große Werte von $a$ verschwindet sie, und das Feld entsteht einfach durch Superposition der Wirkungen einzelner Zylinder.

Es sei noch bemerkt, daB sich die Wirkung der Zylinder aufeinander leicht interpretieren läBt, falls ihr Abstand hinreichend groB ist. Für groBe Werte von $a$ ist nämlich

$$
Q_{n}\left(k_{1} a\right)=-i^{n+1} \sqrt{\frac{\pi}{2 k_{1} a}} e^{i\left(\frac{\pi}{4}-k_{1} a\right)}=i^{n} Q_{0}\left(k_{1} a\right),
$$

und aus den Gleichungen (14) folgt dann

$$
\begin{aligned}
& a_{n 0}=Q_{n}\left(k_{1} a\right), \\
& a_{n m}=2 i^{n+m} Q_{0}\left(k_{1} a\right)=2 i^{m} Q_{n}\left(k_{1} a\right), \\
& b_{n m}=0 .
\end{aligned}
$$

Führt man diese Werte in die Gleichungen (17) und (17') ein, so erhält man für die Koeffizienten $B_{n}$ ganz dieselben Gleichungen wie im Falle der Beugung an einem einzigen Zylinder. Es ist hier $B_{2 m}=0$. In den Gleichungen (16) und (16) darf man zuerst in diejenigen Glieder, in denen die Koeffizienten $a_{m n}$ auftreten, statt $A_{n}$ annähernd richtige Werte einführen, die man erbält, wenn man $a_{m n}=0$ setzt, und welche auch dem Falle eines einzigen Zylinders entsprechen. Wir bezeichnen sie mit $A_{n}$; es ist $A_{2 m+1}=0$. Setzt man nun der Kürze halber (26)

$$
A_{0} Q_{0}+\bar{A}_{2} Q_{2}+A_{4} Q_{4}+\ldots=M
$$

so lauten die Gleichungen (16) und $\left(16^{\prime}\right)$

$$
\begin{aligned}
& A_{0} Q_{0}\left(p_{1}\right)+M J_{0}\left(p_{1}\right)+J_{0}\left(p_{1}\right)=C_{0} J_{0}\left(p_{2}\right) \text {, } \\
& A_{1} Q_{1}\left(p_{1}\right)-2 i M J_{1}\left(p_{1}\right) \quad=C_{1} J_{1}\left(p_{2}\right) \text {, } \\
& A_{2} Q_{2}\left(p_{1}\right)+2 i^{2} M J_{2}\left(p_{1}\right)+2 J_{2}\left(p_{1}\right)=C_{2} J_{2}\left(p_{2}\right) \text {, } \\
& A_{0} Q_{0}^{\prime}\left(p_{1}\right)+M J_{0}^{\prime}\left(p_{1}\right)+J_{0}^{\prime}\left(p_{1}\right)=C_{0} \eta J_{0}^{\prime}\left(p_{2}\right) \text {, } \\
& A_{1} Q_{1}^{\prime}\left(p_{1}\right)-2 i M J_{1}^{\prime}\left(p_{1}\right) \quad=C_{1} \eta J_{1}^{\prime}\left(p_{2}\right) \text {, } \\
& A_{2} Q_{2}{ }^{\prime}\left(p_{1}\right)+2 i^{2} M J_{2}^{\prime}\left(p_{1}\right)+2 J_{2}^{\prime}\left(p_{1}\right)=C_{2} \eta J_{2}^{\prime}\left(p_{2}\right) \text {, }
\end{aligned}
$$


Dieselben Gleichungen würde man auch erhalten, wenn man voraussetzte, daB die Beugung an einem einzigen Zylinder entsteht, daB aber neben der einfallenden Welle, die sich in der Richtung der negativen $y$-Achse bewegt, noch eine andere einfallende Welle auftritt, die durch den Ausdruck

$$
M e^{-i k_{1} r^{\prime} \cos \varphi^{\prime}}=M e^{-i k_{1}\left(x-\frac{a}{2}\right)}
$$

gegeben wäre. Diese Welle pflanzt sich in der Richtung der zunehmenden $x$ fort. Aus der Gleichung (26) sieht man, daB ihre Amplitude proportional mit $a^{-1 / 2}$ ist, und daB ihr periodischer Teil gleich

$$
e^{i\left[\frac{\pi}{4}-k,\left(\frac{a}{2}+x\right)\right]}
$$

ist. Es ist also die vom linken Zylinder ausgehende Störungswelle.

Wir gehen jetzt zur Betrachtung der Beugung an drei parallelen, äquidistanten Zylindern über. Es seien $r, \varphi$ die Polarkoordinaten des Aufpunktes, in bezug auf den Mittelpunkt des mittleren Zylinders, $r^{\prime}, \varphi^{\prime}$ bzw. $r^{\prime \prime}, \varphi^{\prime \prime}$ die Polarkoordinaten des Aufpunktes, auf die Mittelpunkte beider äuBeren Zylinder bezogen. Man erhält dann für die resultierende elektrische Kraft im AuBenraum unter Berücksichtigung der Symmetrieverhältnisse folgenden Ausdruck:

$$
\left\{\begin{aligned}
\tilde{E}_{1} & =e^{i\left(\omega t+k_{1} y\right)}+e^{i \omega t} \sum_{n}^{0, \infty}\left(A_{2 n} Q_{2 n}\left(k_{1} r\right) \cos 2 n \varphi\right. \\
& \left.+B_{2 n+1} Q_{2 n+1}\left(k_{1} r\right) \sin (2 n+1) \varphi\right) \\
& +e^{i \omega t} \sum_{n}^{0, \infty} A_{n}^{\prime}\left(Q_{n}\left(k_{1} r^{\prime}\right) \cos n \varphi^{\prime}+(-1)^{n} Q_{n}\left(k_{1} r^{\prime \prime}\right) \cos n \varphi^{\prime \prime}\right) \\
& +e^{i \omega t} \sum_{n}^{1, \infty} B_{n}^{\prime}\left(Q_{n}\left(k_{1} r^{\prime}\right) \sin n \varphi^{\prime}+(-1)^{n-1} Q_{n}\left(k_{1} r^{\prime \prime}\right) \sin n \varphi^{\prime \prime}\right)
\end{aligned}\right.
$$

für die elektrische Kraft im Innern des mittleren Zylinders folgt dann:

$$
\left\{\begin{aligned}
E_{2} & =e^{i \omega t} \sum_{n}^{0 \cdot \infty}\left(C_{2 n} J_{2 n}\left(k_{2} r\right) \cos 2 n \varphi\right. \\
& \left.+D_{2 n+1} J_{2 n+1}\left(k_{2} r\right) \sin (2 n+1) \varphi\right),
\end{aligned}\right.
$$


endlich für die elektrische Kraft im Innern der beiden äuBeren Zylinder bekommt man:

$$
\left\{\begin{array}{l}
F_{2}^{\prime}=e^{i \omega t} \sum_{0}^{0, \infty}\left(C_{n}^{\prime} \cos n \varphi^{\prime}+D_{n}^{\prime} \sin n \varphi^{\prime}\right) J_{n}\left(k_{2} r^{\prime}\right), \\
\varepsilon_{2}^{\prime \prime}=e^{i \omega t} \sum_{0}^{0, \infty}(-1)^{n}\left(C_{n}^{\prime} \cos n \varphi^{\prime \prime}-D_{n}^{\prime} \sin n \varphi^{\prime \prime}\right) J_{n}\left(k_{2} r^{\prime \prime}\right) .
\end{array}\right.
$$

Die Grenzbedingungen sind an der Oberfläche des mittleren Zylinders und eines der beiden äußeren, z. B. des rechten, zu erfüllen. Man muB also zuerst den Ausdruck (27) in eine Fouriersche Reihe, die nach dem Kosinus und Sinus des vielfachen $\varphi$ bzw. $\varphi^{\prime}$ fortschreitet, umwandeln. Dies läBt sich leicht nach der oben angedeuteten Weise durchführen; man erhält

$$
\begin{aligned}
Q_{n}\left(k_{1} r^{\prime}\right) \cos n \varphi^{\prime} & +(-1)^{n} \quad Q_{n}\left(k_{1} r^{\prime \prime}\right) \cos n \varphi^{\prime \prime} \\
& =2(-1)^{n} \quad \sum_{m}^{0, \infty} a_{n, 2 m} \quad J_{2 m} \quad\left(k_{1} r\right) \cos 2 m \varphi,
\end{aligned}
$$

$Q_{n}\left(k_{1} r^{\prime}\right) \sin n \varphi^{\prime}+(-1)^{n-1} Q_{n}\left(k_{1} r^{\prime \prime}\right) \sin n \varphi^{\prime \prime}$

$$
=2(-1)^{n-1} \sum_{m}^{0, \infty} b_{n, 2 m+1} J_{2 m+1}\left(k_{1} r\right) \sin (2 m+1) \varphi,
$$

$Q_{n}\left(k_{1} r\right) \cos n \varphi=\sum_{m}^{0, \infty}(-1)^{m} \quad a_{n m} J_{m}\left(k_{1} r^{\prime}\right) \cos m \varphi^{\prime}$,

$Q_{n}\left(k_{1} r\right) \sin n \varphi=\sum_{m}^{1, \infty}(-1)^{m-1} b_{n m} J_{m}\left(k_{1} r^{\prime}\right) \sin m \varphi^{\prime}$,

$Q_{n}\left(k_{1} r^{\prime \prime}\right) \cos n \varphi^{\prime \prime}=\sum_{m}^{0, \infty}(-1) \quad a_{n m} J_{m}\left(k_{1} r^{\prime}\right) \cos m \varphi^{\prime}$,

$Q_{n}\left(k_{1} r^{\prime \prime}\right) \sin n \varphi^{\prime \prime}=\sum_{m}^{1, \infty}(-1)^{m-1} \bar{b}_{n m} J_{m}\left(k_{1} r^{\prime}\right) \sin m \varphi^{\prime}$,

wo die GröBen $a_{n m}, b_{n m}$ durch die Gleichungen (14) definiert sind; weiter gilt:

$$
\left\{\begin{array}{l}
\bar{a}_{n 0}=Q_{n}\left(2 k_{1} a\right), \\
\bar{a}_{n m}=Q_{n+m}\left(2 k_{1} a\right)+(-1)^{m} \quad Q_{n-m}\left(2 k_{1} a\right), \\
\bar{b}_{n m}=Q_{n+m}\left(2 k_{1} a\right)+(-1)^{m-1} Q_{n-m}\left(2 k_{1} a\right) .
\end{array}\right.
$$

Stellt man also die Grenzbedingungen (6) und (6') für die Oberfläche eines jeden Zylinders auf, so bekommt man, wie 
im vorigen Falle, zwei voneinander unabhängige Systeme linearer Beziehungen zwischen den Koeffizienten $A_{2 n}, B_{2 n+1}$ usw. Nach Elimination von $C_{2 n}, D_{2 n+1}, C_{n}^{\prime}$ und $D_{n}^{\prime}$ lauten die Gleichungen:

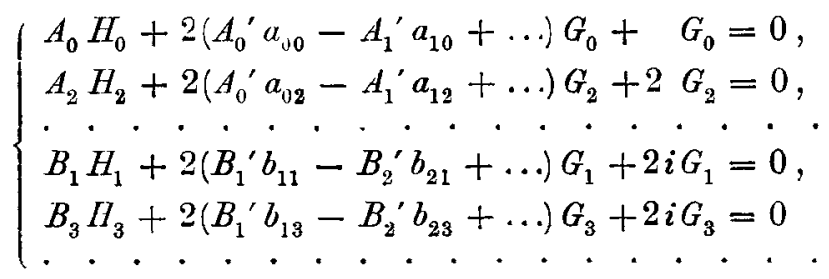

und

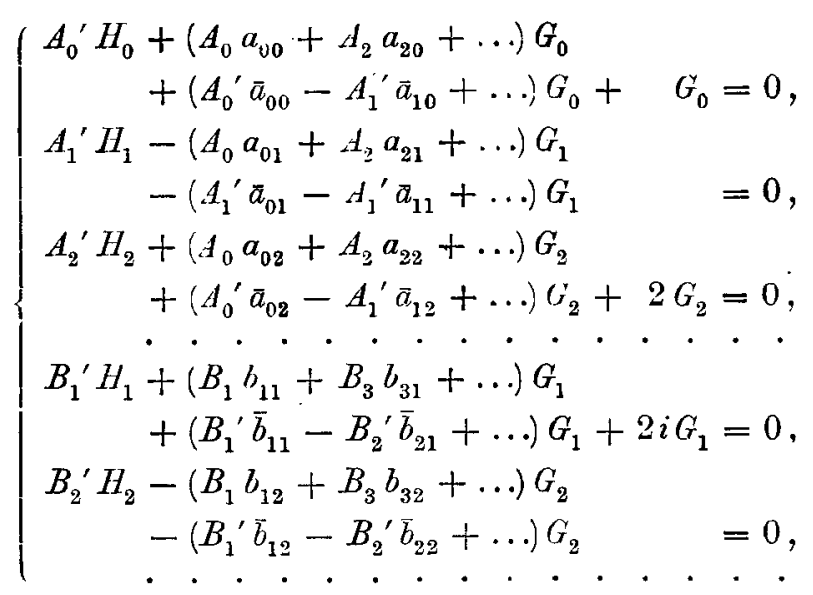

wo $G_{n}$ und $H_{n}$ durch die Gleichungen (18) bestimmt sind.

Ü̉ber die Auflösung der Gleichungen (29) und (29') gilt nun dasselbe, was schon über die Gleichungen (16) und $\left(16^{\prime}\right)$ gesagt wurde; auch die Diskussion des Feldes dreier Zylinder in den Punkten, deren Entfernung von den Zylindern hinreichend groß ist, führt zu ähnlichen Schlüssen wie früher. Wir wollen also gleich zu dem einfachen Falle übergehen, wo die Zylinder sehr dünn sind; man darf dann von allen in den Gleichungen (29) und (29') auftretenden Koeffizienten nur $A_{0}$ und $A_{0}{ }^{\prime}$ behalten. Man erhält:

$$
\begin{aligned}
A_{0} H_{0}+2 A_{0}^{\prime} a_{00} G_{0}+G_{0} & =0, \\
A_{0} a_{00} G_{0}+A_{0}^{\prime}\left(H_{0}+\bar{a}_{00} G_{0}\right)+G_{0} & =0,
\end{aligned}
$$


woraus folgt

wo gesetzt wurde:

$$
\begin{aligned}
& A_{0}=-\frac{M}{\frac{H_{0}}{G_{0}} M+2 a_{00} N}, \\
& A_{0}^{\prime}=-\frac{N}{\frac{H_{0}}{\theta_{0}}+2 a_{00} N},
\end{aligned}
$$

$$
M=\frac{H_{0}}{G_{0}}+\bar{a}_{00}-2 a_{00}, \quad N=\frac{I I_{0}}{\Theta_{0}}-a_{00}
$$

Das Feld im Außenraum ist nach Gleicbung (27) durch den folgenden Ausdruck gegeben:

$$
\left\{\begin{array}{c}
F_{1}=e^{i\left(\omega t+k_{1} y\right)}+t^{i \omega t}\left(A_{0} Q_{0}\left(k_{1} r\right)+A_{0}{ }^{\prime} Q_{0}\left(k_{1} r^{\prime}\right)\right. \\
\left.+A_{0}^{\prime} Q_{0}\left(k_{1} r^{\prime}\right)\right)=R \epsilon^{i \omega t}
\end{array}\right.
$$

Setzt man nun

$$
R=P+i Q \text {, }
$$

wo $P$ und $Q$ reell sind, so folgt für den zeitlichen Mittelwert der Feldintensität:

$$
\bar{F}_{1}^{2}=\frac{1}{2}\left(P^{2}+Q^{2}\right) \text {. }
$$

Mit Hilfe dieser Formeln ist nun die mittlere Feldintensität dreier Kupferdrähte von $1 \mathrm{~mm}$ Durchmesser, für verschiedene Abstände $a$, berechnet. Die Wellenlänge $\lambda=31,4 \mathrm{~cm}$, als Aufpunkte sind zwei Punkte der Symmetrieebene, der eine $10 \mathrm{~cm}$, der andere $40 \mathrm{~cm}$ hinter dem mittleren Zylinder liegend (also $y=-10 \mathrm{~cm}$ bzw. $y=-40 \mathrm{~cm}$ ), gewählt. Die Leitfähigkeit $\sigma=5,25 \cdot 10^{17}$ elstat. Einh., woraus folgt

$$
p_{2}=234(1-i), \quad \frac{1}{\eta}=2,13 \cdot 10^{-5}(1+i) .
$$

$\mathrm{Da} p_{2}$ einen sehr groBen Wert hat, dessen imaginärer Teil negativ ist, so darf man mit hinreichender Genauigkeit setzen

$$
\frac{J_{0}^{\prime}\left(p_{2}\right)}{J_{v}^{\prime}\left(p_{2}\right)}=i-\frac{1}{2} p_{2}=i-1,1 \cdot 10^{-3}(1+i) \text {. }
$$

Die Werte der Feldintensität sind auf die mittlere Intensität des störungsfreien Feldes bezogen (d. h. der Wert von $\bar{F}_{1}^{2}$ ist mit 2 multipliziert) und in der dritten bzw. fünften Kolonne der beigegebenen Tab. I angegeben. Zum Vergleich sind in der zweiten und vierten Kolonnel die Werte der mittleren Feldintensität zweier Kupferdrähte von demselben 
Durchmesser und für gleiche Wellenlänge angeführt. Die Aufpunkte liegen jetzt hinter einem der Drähte (es ist also z. B. $\left.\varphi^{\prime}=-\pi / 2\right)$ in denselben Entfernungen wie früher.

Tabelle I.

$\varrho=0,1 \mathrm{~cm}, \lambda=31,4 \mathrm{~cm}, \sigma=5,25 \cdot 10^{17}$ elstat. Einh.

\begin{tabular}{l||c|c|c|c}
\hline \multirow{2}{*}{$a$} & \multicolumn{2}{|c|}{$y=-10 \mathrm{~cm}$} & \multicolumn{2}{c}{$y=-40 \mathrm{~cm}$} \\
\cline { 2 - 5 } & Zwei Drähte & Drei Drähte & Zwei Drähte & Drei Drähte \\
\hline \hline $1 \mathrm{~cm}$ & 0,589 & 0,507 & 0,780 & 0,731 \\
3 & 0,526 & 0,374 & 0,737 & 0,640 \\
5 & 0,497 & 0,294 & 0,708 & 0,561 \\
7,5 & 0,505 & 0,331 & 0,683 & 0,507 \\
10 & 0,579 & 0,555 & 0,678 & 0,542 \\
15 & 0,845 & 0,998 & 0,756 & 0,697 \\
20 & 0,912 & 1,240 & 0,862 & 0,874 \\
25 & 0,862 & 1,040 & 0,937 & 1,048 \\
30 & 0,721 & 0,746 & 0,970 & 1,089 \\
40 & 0,553 & 0,413 & 0,962 & 1,092 \\
50 & 0,829 & 0,956 & 0,985 & 0,721 \\
$\infty$ & 0,708 & 0,708 & 0,849 & 0,848
\end{tabular}

Man sieht, daB die Schattenwirkung eines einzelnen Drahtes $(a=\infty)$ durch die Anwesenheit anderer Drähte nicht immer verstärkt erscheint; manchmal kann dieselbe sogar aufgehoben werden, so daB dann die Feldintensität hinter dem Drahte gröBer ist als diejenige des ungestörten Feldes. Freilich tritt dies nur in den Punkten auf, die ziemlich nahe an den Drähten liegen. Ist dagegen die Entfernung des Aufpunktes hinreichend gro $B$ gegen die Wellenlänge und gegen den $A b-$ stand der Drähte, so wird das Feld immer geschwächt; wie es sich leicht auf Grund der Gleichung (30) zeigen läBt, wenn man in derselben die Besselschen Funktionen durch die asymptotischen Werte darstellt und bedenkt, daB sowohl der reelle als auch der imaginäre Teil von $A_{0}$ und $A_{0}^{\prime}$ immer negativ ist.

Die Schattenwirkung der Drähte erreicht ihr Maximum für einen gewissen Wert von $a$, der von der Lage des Aufpunktes abhängt; rücken die Drähte näher aneinander, so wird 
das Feld verštärkt. Mit wachsendem $a$ ändert sich das Feld periodisch; für sehr große Werte von $a$ ist die Periode gleich $\lambda$.

2. Die elektrische Kraft ist senkrecht zu den Zylinderachsen.

Dieser Fall ist theoretisch und experimentell weniger interessant als der vorige und wir wollen ihn nur kurz betrachten. Von den elektrischen Kraftkomponenten treten hier nur $\mathbb{F}_{r}$ und $\mathbb{F}_{\varphi}$ auf, von den magnetischen kommt nur die den Zylinderachsen parallele Komponente $\mathfrak{F}_{z}$ vor. Da auBerdem alles von $z$ unabhängig ist, so lauten die Maxwellschen Gleichungen einfach:

$$
\left\{\begin{aligned}
\frac{\mu}{c} \frac{\partial \mathfrak{S}_{z}}{\partial t} & =-\frac{1}{r} \frac{\partial}{\partial r}\left(r \mathfrak{E}_{\varphi}\right)+\frac{1}{r} \frac{\partial \mathfrak{E}_{r}}{\partial \varphi}, \\
\frac{1}{c}\left(\varepsilon \frac{\partial \mathfrak{E}_{r}}{\partial t}+4 \pi \sigma \mathfrak{E}_{r}\right) & =\frac{1}{r} \frac{\partial \mathfrak{G}_{z}}{\partial \varphi}, \\
\frac{1}{c}\left(\varepsilon \frac{\partial \mathfrak{E}_{\varphi}}{\partial t}+4 \pi \sigma \mathfrak{E}_{\varphi}\right) & =-\frac{\partial \mathfrak{W}_{z}}{\partial r} .
\end{aligned}\right.
$$

Aus denselben erhält man in bekannter Weise folgende Differentialgleichungen für $\mathfrak{S}-$ wie wir jetzt kurz statt $\mathfrak{S}_{z}$ schreiben wollen:

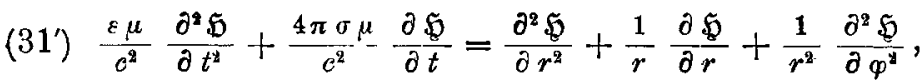

eine Gleichung, die mit der Gleichung (1') für $\mathbb{E}$ vollkommen identisch ist und für deren Integral man wieder den Ausdruck (2) setzen darf. Ebenso ist die resultierende magnetische Kraft im Außenraum und im Innenraum durch die Ausdrücke (4) und (5) gegeben; es ist also

$$
\begin{aligned}
\mathfrak{W}_{1} & =e^{i\left(\omega t+k_{2} y\right)}+e^{i \omega t} \sum_{h}^{1, N} \sum_{n}^{0, \infty}\left(A_{h n} \cos n \varphi_{h}+B_{h n} \sin n \varphi_{h}\right) Q_{n}\left(k_{1} r_{h}\right), \\
\mathfrak{W}_{2 h} & =e^{i \omega t} \sum_{n}^{0, \infty}\left(C_{h n} \cos n \varphi_{h}+D_{h n} \sin n \varphi_{h}\right) J_{n}\left(k_{2} r_{h}\right) .
\end{aligned}
$$
lauten:

Die Grenzbedingungen an der Oberfläche des $h^{\text {ten }}$ Zylinders

$$
\left\{\begin{aligned}
\mathfrak{F}_{1} & =\mathfrak{S}_{2 h} \\
\left(\mathfrak{F}_{\varphi}\right)_{1} & =\left(\mathfrak{E}_{\varphi}\right)_{2 h}
\end{aligned}\right.
$$

statt der letzten Gleichung darf man wieder schreiben

$$
\frac{\partial \mathfrak{W}_{1}}{\partial r_{h}}=\frac{\nu_{1}}{\nu_{2}} \frac{\partial \mathfrak{W}_{2 h}}{\partial r_{h}}
$$


wo gesetzt wurde:

$$
\nu=4 \pi \sigma+i \varepsilon \omega .
$$

Mit Berücksichtigung der Gleichung (3) erbält man auch:

$$
v=\frac{i e^{2}}{\omega \mu} k^{2} \text {. }
$$

Man sieht, daB sich die Gleichungen (32) und (32') von den im vorigen Falle geltenden Grenzbedingungen (6) und (6') nur dadurch unterscheiden, $\mathrm{da} B$ in denselben $\nu_{1}$ und $\nu_{2}$ mit $\mu_{1}$ und $\mu_{2}$ vertauscht erscheint. Daraus folgt weiter, daB die dort entwickelten Gleichungen auch hier bestehen, nur muB der Wert von $\eta$, der durch die Gleichung (15) bestimmt ist, geändert werden; es ist nämlich jetzt

$$
\eta=\frac{k_{2}}{k_{1}} \frac{\nu_{1}}{\nu_{2}}=\frac{k_{1}}{k_{2}} \frac{\mu_{2}}{\mu_{1}} \text {. }
$$

Handelt es sich um dielektrische Zylinder, so ist

$$
\eta=\frac{\mu_{2}}{\sqrt{\varepsilon}} ;
$$

sind die Zylinder gute Leiter, so hat man:

$\eta$ ist also hier sehr klein.

$$
\eta=\sqrt{\frac{c \mu_{2}}{\sigma \lambda}} \frac{1+i}{2},
$$

Wir wollen uns der Einfachheit halber auf den Fall zweier Zylinder beschränken und gleich voraussetzen, daB beide Zylinder sehr dünn sind. Sind nun die Zylinder dielektrisch und darf man $p_{2}=p_{1} \sqrt{\varepsilon}$ auch als klein betrachten, so folgt aus den Gleichungen (18), daB $G_{0}$ von der Ordnung $p_{1}{ }^{3}$ ist, alle anderen $G_{n}$ sind von der Ordnung $p_{1}{ }^{2 n-1}$, endlich alle $H_{n}$ sind von der Ordnung $1 / p_{1}$. Aus den Gleichungen (19) und $\left(19^{\prime}\right)$ sieht man dann, daB man in erster Annäherung alle $A_{n}$ gleich Null setzen darf; weiter ist

$$
B_{1}=i p_{1}^{2} \frac{\varepsilon-1}{\varepsilon+1}\left(1+b_{11} \frac{p_{1}^{2}}{2} \frac{\varepsilon-1}{\varepsilon+1}\right) \text {, }
$$

alle übrigen $B_{n}$ darf man wieder vernachlässigen. Die Wirkung der Zylinder aufeinander ist also wieder gering.

Sind die Zylinder gute Leiter, so darf man in den Gleichungen (18) die mit $\eta$ behafteten Glieder streichen; dann folgt, daB das Verhältnis $G_{n} / H_{n}$ von der Ordnung $p_{1}{ }^{2 n}, G_{0} / H_{0}$ von der Ordnung $p_{1}^{2}$ ist. Es sind also die Koeffizienten $A_{0}$ 
und $B_{1}$ von derselben 0 rdnung, alle übrigen $A_{n}$ und $B_{n}$ darf man. vernachlässigen. Man erhält aus den Gleichungen (19) und (19').

$$
\begin{aligned}
& A_{0}=-\frac{p_{1}^{2}}{2}\left(1-a_{00} \frac{p_{1}^{2}}{2}\right), \\
& B_{1}=i p_{1}{ }^{2} \quad\left(1+b_{11} \frac{p_{1}^{2}}{2}\right) .
\end{aligned}
$$

Vergleicht man diese Gleichungen mit der Gleichung $\left(\mathbf{2 5}^{\prime \prime}\right)$, die dem Falle entspricht, daB die elektrische Kraft der einfallenden Welle parallel zu den Zylinderachsen verläuft, so sieht man gleich, daB, wenn die elektrische Kraft senkrecht zu den Zylinderachsen gerichtet ist, die Wirkung der Zylinder aufeinander viel geringer und auch die durch die Zylinder hervorgerufene Störung des Feldes bedeutend kJeiner, ja kaum bemerkbar ist, sobald der Aufpunkt nicht allzu nahe an der Oberfläche der Drähte liegt. Es ist auch ohne weiteres klar, daB. dasselbe auch bei der Beugung an drei Zylindern gültig bleibt.

Daraus folgt nun ein interessantes Resultat. Bei seinen bekannten Gitterversuchen fand H. Hertz, daB ein Gitter aus zylindrischen Metallstäben die elektrische Energie der auffallenden Welle in verschiedener Weise beeinflubt, je nachdem die elektrische Kraft zu den Stäben parallel oder senkrecht gerichtet ist. Im ersten Falle wird das Feld nahezu völlig. abgeschirmt, im zweiten Falle dagegen läBt das Gitter fast die ganze einfallende Energie durch. Ähnliche Verhältnisse findet man auch, falls die Beugung an einem einzigen Metalldrahte vor sich geht; das "Durchlässigkeitsvermögen" eines solchen Drahtes ist für die Punkte, die hinter ihm liegen, kleiner, wenn die elektrische Kraft der einfallenden Welle parallel mit dem Drahte verläuft als im anderen Falle wenigstens solange das Verhältnis $\varrho / \lambda$ hinreichend klein bleibt. Wir wollen diesen Effekt mit Schaefer den Hertzeffeht nennen. Aus den in Tab. I angeführten Zahlen sieht man nun, daB im Falle der Beugung an zwei oder drei parallelen Metalldrähten das dem Hertzeffekt entgegengesetzte Phänomen statt. finden kann; unter Umständen ist hier nämlich die mittlere Feldintensität in den Punkten, die hinter einem der Drähte liegen, bedeutend gröBer, wenn die elektrische Kraft parallel zu den Drähten verläuft. Dieser Effekt wurde von Schaefer Duboiseffekt genannt. Man sieht aber auch, daB das Auftreten 
des Duboiseffektes nur auf die Punkte beschränkt ist, welche ziemlich nahe an den Drähten liegen; für weiter entfernte Punkte läBt sich nur der Hertzeffekt erwarten, wie ihn auch Schaefer und Reichel) für Gitter, deren Gitterkonstante grob ist, gefunden haben.

3. Zwei Zylinder hintereinander. Die elektrische Kraft ist parallel zu den Zylinderachsen.

Wir wollen jetzt die Beugung an zwei hintereinander stehenden, parallelen Zylindern untersuchen. Die Fortpflanzungsrichtung der einfallenden Welle ist also jetzt parallel zur Ebene, welche durch die beiden Zylinderachsen bestimmt ist. Beide Zylinder dürfen verschieden sein. Wir werden uns nur anf den Fall beschränken, daB die elektrische Kraft der einfallenden Welle parallel zu den Zylinderachsen verläuft.

Macht man die Ebene beider Zylinderachsen wieder zur $x \boldsymbol{z}$-Ebene, so fällt die Fortpflanzungsgeschwindigkeit der einfallenden Welle in die $x$-Achse, und setzt man voraus, daB sich dieselbe in der Richtung der abnehmenden $x$ bewegt, so hat man:

oder auch (vgl. Fig. 2)

$$
\dot{v}_{1 e}=e^{i\left(\omega t+k_{1} x\right)},
$$

$$
E_{1 e}=e^{i\left(\omega t+k_{1} r^{\prime} \cos \varphi^{\prime}+k_{1} \frac{a}{2}\right)}=e^{i\left(\omega t+k_{1} r^{\prime \prime} \cos \varphi^{\prime \prime}-k_{1} \frac{a}{2}\right)} .
$$

Da die $x z$-Ebene offenbar eine Symmetrieebene ist, so folgt für die vom vorderen Zylinder ausgehende Störungswelle:

$$
\xi_{1 r}^{\prime}=e^{i \omega t} \sum_{n}^{0, \infty} A_{n} Q_{n}\left(k_{1} r^{\prime}\right) \cos n \varphi^{\prime}
$$

ähnlich bekommt man für die vom hinteren Zylinder ausgehende Störungswelle:

$$
\mathbb{E}_{1 r}^{\prime \prime}=e^{i \omega t} \sum_{n}^{0, \infty} B_{n} Q_{n}\left(k_{1} r^{\prime \prime}\right) \cos n \varphi^{\prime \prime},
$$

so daB die resultierende elektrische Kraft im Außenraum durch den folgenden Ausdruck gegeben erscheint:

$$
\left\{\begin{aligned}
F_{1}= & e^{i\left(\omega t+k_{1} x\right)} \\
& +c^{i \omega t} \sum_{n}^{\infty \infty}\left(A_{n} Q_{n}\left(k_{1} r^{\prime}\right) \cos n \varphi^{\prime}+B_{n} Q_{n}\left(k_{1} r^{\prime \prime}\right) \cos n \varphi^{\prime \prime}\right) .
\end{aligned}\right.
$$

1) Cl. Schaefer u. F. Reiche, l. c. 
Für die elektrische Kraft im Innern des vorderen Zylinders setzen wir:

$$
\xi_{2}{ }^{\prime}=e^{i \omega t} \sum_{n}^{0, \infty} C_{n} \partial_{n}\left(k_{2}{ }^{\prime} r^{\prime}\right) \cos n \varphi^{\prime}
$$

endlich folgt für die elektrische Kraft im Innern des hinteren Zylinders der Ausdruck:

$$
F_{2}^{\prime \prime}=e^{i \omega t} \sum_{n}^{0, \infty} D_{n} J_{n}\left(k_{2}^{\prime \prime} r^{\prime \prime}\right) \cos n \varphi^{\prime \prime}
$$

Die Grenzbedingungen sind durch die Gleichungen (6) und $\left(6^{\prime}\right)$ gegeben; man muB sie aber für die Oberfläche eines jeden Zylinders gesondert aufstellen. Was den vorderen Zylinder betrifft, so ist die Rechnung ganz dieselbe, wie sie schon für den Fall zweier nebeneinander stehender Zylinder durchgeführt wurde, und braucht nicht wiederholt zu werden. Mit Hilfe der Gleichung

$$
e^{i k_{1} r^{\prime} \cos \varphi^{\prime}}=J_{0}\left(k_{1} r^{\prime}\right)+2 \sum_{n}^{1, \infty} i^{n} J_{n}\left(k_{1} r^{\prime}\right) \cos n \varphi^{\prime}
$$

und der Gleichungen (13) gelangt man zu folgenden Beziehungen:

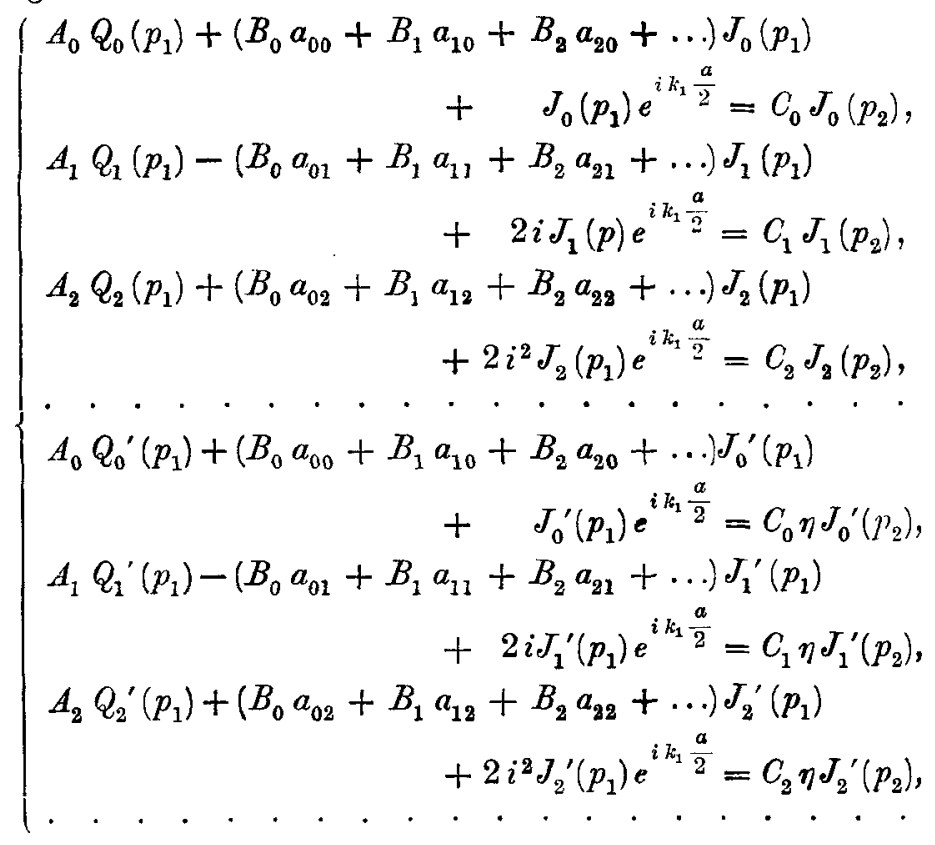


wo gesetzt wurde:

$$
\left\{\begin{array}{l}
k_{1} \varrho^{\prime}=p_{1}, \quad k_{2}^{\prime} \varrho^{\prime}=p_{2}, \\
\frac{\mu_{1}}{\mu_{2}^{\prime}} \frac{k_{2}^{\prime}}{k_{1}}=\frac{\mu_{1}}{\mu_{2}^{\prime}} \frac{p_{2}}{p_{1}}=\eta ;
\end{array}\right.
$$

$\rho^{\prime}$ bedeutet den Radius des vorderen Zylinders. Die Koeffizienten $a_{m n}$ sind durch die Gleichungen (14) gegeben.

Geht man zum hinteren Zylinder über, so hat man zuerst den Ausdruck (34) für $\mathfrak{E}_{1}$ in eine Fouriersche, nach dem Kosinus und Sinus des vielfachen $\varphi^{\prime \prime}$ fortschreitende Reihe umzuformen. In derselben Weise wie früher erhält man hier:

$$
Q_{n}\left(k_{1} r^{\prime}\right) \cos n \varphi^{\prime}=(-1)^{n} \sum_{m}^{0, \infty} a_{n m} J_{m}\left(k_{1} r^{\prime \prime}\right) \cos m \varphi^{\prime \prime},
$$

so daB die Grenzbedingungen (6) und (6') folgende Gleichungen liefern:

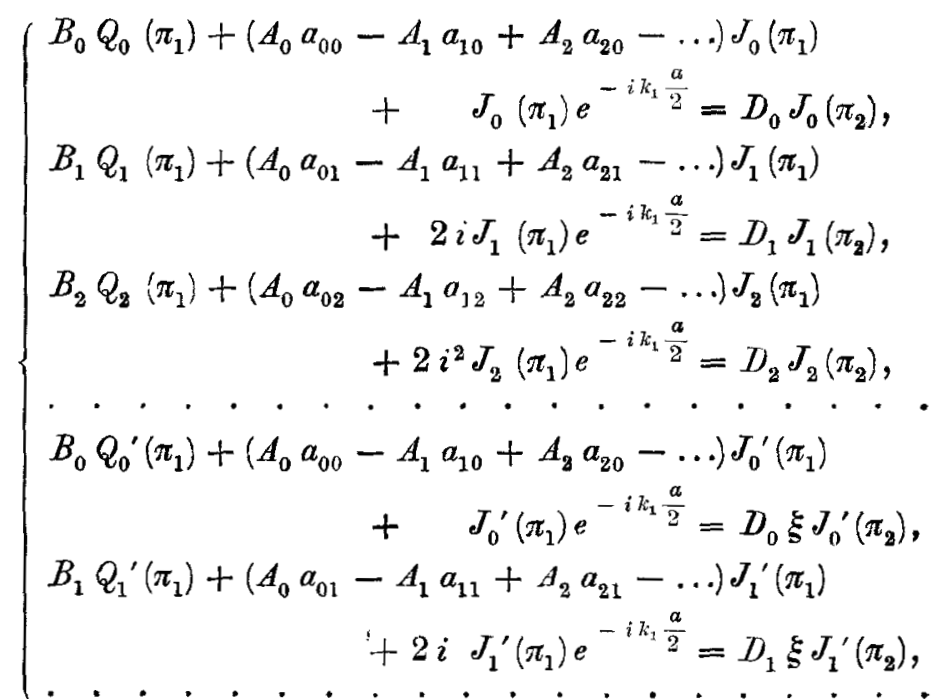

wo gesetzt wurde:

$$
\left\{\begin{array}{c}
k_{1} \varrho^{\prime \prime}=\pi_{1}, \quad k_{2}^{\prime \prime} \varrho^{\prime \prime}=\pi_{2}, \\
\frac{\mu_{1}}{\mu_{2}^{\prime \prime}} \frac{\pi_{2}}{\pi_{1}}=\xi
\end{array}\right.
$$

$\rho^{\prime \prime}$ bedeutet den Radius des hinteren Zylinders. 
Wir wollen nun die Gleichungen (36) und (37) dazu benutzen, um einige Resultate, zu denen Grossmann ${ }^{1}$ ) bei seinen Messungen der Beugung an einem Zylinder gelangte, ünd die mit der Theorie nicht übereinstimmen, zu erklären. Zur Erzeugung der primären ebenen Wellen diente ihm ein Hertzscher Erreger, der in der Brennlinie eines parabolischen Spiegels aufgestellt war. Die Wellen fielen auf ein zylindrisches, dünnwandiges Glasrohr, das mit Wasser gefüllt war, und die Strahlung wurde mit einem Klemencičschen Thermoelement gemessen, das auf den Erreger abgestimmt war. Die Messungen wurden in der Weise durchgeführt, daB zuerst die freie Strahlung gemessen wurde. Es wurde dann in den Strahlengang ein Zylinder gebracht, und die Strahlung wieder bestimmt; das Verhältnis beider so erhaltenen Werte wurde dann mit dem theoretischen Werte verglichen.

Die Übereinstimmung mit der Theorie war so genau, wie es sich eben in Anbetracht der Schwierigkeiten, mit welchen die Messungen verbunden waren, erwarten lieb; nur in einem Punkte zeigte sich ein wesentlicher Unterschied. Den theoretischen Resultaten zufolge sollen in den hinter dem Zylinder liegenden Punkten keine Interferenzstreifen entsteben; wenn die Entfernung des Aufpunktes ron dem Zylinder wächst, so soll die Feldintensität entweder fortwährend abnehmen, bis sie den ursprünglichen Wert (ohne Zylinder) erreicht, oder sie soll fortwährend zunehmen, oder endlich nimmt sie zuerst ab, erreicht ein Minimum, und dann nähert sie sich steigend allmählich dem der freien Strahlung entsprechenden Werte. Die Messungen ergaben dagegen in einigen Fällen periodische Schwankungen der Intensität, die mit wachsendem Abstand vom Zylinder abnahmen; die Periode war angenähert $\lambda / 2$. Aus dem Umstande, daB diese Schwankungen nur dann auftraten, wenn der Radius des Zylinders verhältnismäBig groB und die Entfernung des Aufpunktes vom Zylinder gegen die Wellenlänge klein war, erklärten die Autoren ihr Auftreten dadurch, dab das MeBinstrument selbst das zu messende Feld ändert. Im folgenden wollen wir nun zeigen, daB die Theorie wirklich periodische Schwankungen der Feldintensität in den hinter

1) Cl. Schaefer u. F. Grossmann, l. c. 
dem Zylinder liegenden Punkten ergibt, wenn man die Wirkung des Thermoelementes auf den Zylinder berücksichtigt.

$\mathrm{Zu}$ dem $\mathrm{Zwecke}$ denken wir uns das Thermoelement durch einen unendlich langen, sehr dünnen Metalldraht ersetzt. Die Beugung erfolgt an beiden Zylindern; miBt man also die Feldintensität in einem hinter dem Zylinder liegenden Punkte, so hat man den gerade untersuchten Fall. Da der Radius des hinteren Zylinders äuBerst klein ist, so darf man in den Gleichungen (36) und (37) $B_{1}, B_{2}, \ldots$ gegen $B_{0}$ vernachlässigen. A us den Gleichungen (36) folgt dann:

$$
A_{n}=\alpha_{n}+\beta_{n} B_{0}
$$

wobei gilt:

$$
\left\{\begin{array}{c}
\alpha_{0}=-\frac{G_{0}}{H_{0}} e^{i k_{1} \frac{a}{2}}, \quad \alpha_{n}=-2 i^{n} \frac{G_{n}}{H_{n}} e^{i k_{1} \frac{a}{2}}, \\
\beta_{n}=(-1)^{n-1} \frac{G_{n}}{H_{n}} a_{0 n}
\end{array}\right.
$$

die Koeftizienten $G_{n}$ und $H_{n}$ sind durch die Gleichungen (18) gegeben. Von den Gleichungen (37) bleiben jetzt nur zwei übrig, die nach der Einführung des Ausdruckes (38) für $A_{n}$ lauten:

$$
\left\{\begin{array}{l}
B_{0}\left(Q_{0}\left(\pi_{1}\right)+\beta J_{0}\left(\pi_{1}\right)\right)+J_{0}\left(\pi_{1}\right)\left(\alpha+e^{-i k_{1} \frac{a}{2}}\right)=D_{0} J_{0}\left(\pi_{2}\right) \\
B_{0}\left(Q_{0}{ }^{\prime}\left(\pi_{1}\right)+\beta J_{0}^{\prime}\left(\pi_{1}\right)\right)+J_{0}^{\prime}\left(\pi_{1}\right)\left(\alpha+e^{-i k_{1} \frac{a}{2}}\right)=D_{0} \xi J_{0}^{\prime}\left(\pi_{2}\right),
\end{array}\right.
$$

wo der Kürze halber gesetzt wurde:

$\left(39^{\prime}\right)\left\{\begin{array}{l}\alpha=\alpha_{0} a_{00}-\alpha_{1} a_{10}+\ldots=\alpha_{0} Q_{0}\left(k_{1} a\right)-\alpha_{1} Q_{1}\left(k_{1} a\right)+\ldots, \\ \beta=\beta_{0} a_{00}-\beta_{1} a_{10}+\ldots=\beta_{0} Q_{0}\left(k_{1} a\right)-\beta_{1} Q_{1}\left(k_{1} a\right)+\ldots\end{array}\right.$

Die Gleichungen (39) vereinfachen sich noch bedeutend, wenn man voraussetzt, daB der Metalldraht so fein ist, daB man nicht nur $\pi_{1}$, sondern auch $\pi_{2}$ als klein betrachten darf. Mit Hilfe der bekannten Formeln für die Werte der Zylinder. funktionen bei kleinen Argumenten erhält man dann:

$$
\left\{\begin{array}{l}
B_{0}\left(\log \frac{2}{\gamma \pi_{1}}+\beta\right)+\alpha+e^{-i k_{1} \frac{a}{2}}=D_{0}, \\
B_{1} \cdot \frac{1}{\pi_{1}}+\frac{\pi_{1}}{2}\left(\alpha+e^{-i k_{1} \frac{a}{2}}\right)=D_{0} \xi \frac{\pi_{9}}{2}
\end{array}\right.
$$


Die Ausschläge des mit dem Thermoelemente verbundenen Galvanometers sind der in demselben während der Zeiteinheit entwickelten Wärmemenge proportional. Ist der Drahtradius sehr klein, so ist der Strom auch bei diesen schnellen Schwingungen über den Querschnitt gleichmäBig verteilt, und die Angaben des Galvanometers dürfen als $\mathrm{MaB}$ für den zeitlichen Mittelwert des Quadrates der Stromintensität, oder auch des Quadrates der im Innern des Drahtes herrschenden elektrischen Kraft betrachtet werden. Für dieselbe folgt nun aus der Gleichung $\left(35^{\prime}\right)$ in der hier benutzten Annäherung:

$$
\mathcal{E}_{2}^{\prime \prime}=D_{0} e^{i \omega t},
$$

wo $D_{0}$ durch die Gleichungen $\left(39^{\prime \prime}\right)$ bestimmt wird. Mit Berücksichtigung der letzten Gleichung $\left(37^{\prime}\right)$, in der wir der Einfachbeit halber $\mu_{1}=\mu_{2}^{\prime \prime}=1$ setzen wollen, erhält man für $D_{0}$ folgenden Ausdruck:

$$
D_{0}=\left(1+\frac{\pi_{2}^{2}}{2}\left(\log \frac{2}{\gamma \pi_{1}}+\beta\right)\right)\left(e^{-i k_{1} \frac{a}{2}}+\alpha\right)
$$

Aus der Gleichung (38) sieht man, daB es die mit $\beta_{n}$ behafteten Glieder sind, die der Rückwirkung des Thermoelementes auf den vorderen Zylinder entsprechen, nach Vernachlässigung derselben bekommt man nämlich für $A_{n}$ denselben Ausdruck, als ob die Beugung nur am vorderen Zylinder erfolgen würde. Der durch die Gleichung $\left(40^{\prime}\right)$ gegebene Wert von $D_{0}$ lautet dann:

$$
\bar{D}_{0}=\left(1+\frac{\pi_{2}^{2}}{2} \log \frac{2}{\gamma \pi_{1}}\right)\left(e^{-i k_{1} \frac{a}{2}}+\alpha\right),
$$

und daraus folgt für die elektrische Kraft im Innern des Drahtes:

$$
\bar{F}_{2}^{\prime \prime}=\bar{D}_{0} e^{i \omega t} \text {. }
$$

Wir wollen diesen Ausdruck den ,theoretischen Wert der Feldintensität" nennen; erfolgt nämlich die Beugung nur am vorderen Zylinder, so ist die Feldintensität in der Entfernung a hinter dem Zylinder gleich:

$$
\begin{aligned}
\left(e^{-i k_{1} \frac{a}{2}}+\alpha_{0} Q_{0}\left(k_{1} a\right)-\alpha_{1} Q_{1}\left(k_{1} a\right)+\ldots\right) e^{i \omega t} & \\
& =\left(e^{-i k_{1} \stackrel{a}{2}}+\alpha\right) e^{i \omega t},
\end{aligned}
$$


und aus den Gleichungen (41) und $\left(41^{\prime}\right)$ sieht man, daB $\bar{F}_{2}^{\prime \prime}$ derselben proportional ist; der Proportionalitätsfaktor hängt nur von den Konstanten des Drahtes und ron der Wellenlänge ab, nicht aber von der Entfernung des Drahtes vom vorderen Zylinder.

Die Messungen dagegen beziehen sich auf den durch die Gleichung (40) gegebenen Wert von $\mathbb{F}_{2}{ }^{\prime \prime}$; für das Verhältnis beider Werte erhält man:

$$
\frac{\mathfrak{F}_{2}^{\prime \prime}}{\bar{F}_{2}^{\prime \prime}}=1+\frac{\pi_{*}^{2}}{2} \beta
$$

Da nun durch die Messungen die zeitlichen Mittelwerte bestimmt werden, so setzen wir:

$$
\frac{\mathbb{F}_{2}^{\prime \prime}}{\vec{F}_{2}^{\prime \prime}}=M+i N
$$

das Verhältnis $q$ zwischen dem beobachteten und dem theoretischen Werte ist dann:

$$
q=M^{2}+N^{2}
$$

Wir wollen diesen Ausdruck noch vereinfachen. Ist der Radius des vorderen Zylinders auch klein gegen die Wellenlänge, so darf man in erster Annäherung setzen:

$$
\beta=\beta_{0} a_{00}=-\frac{G_{0}}{H_{\diamond}} Q_{0}{ }^{2}\left(k_{1} a\right),
$$

und beschränkt man sich auf Punkte, die nicht allzu nahe an der Oberfläche des vorderen Zylinders liegen, so folgt:

Es sei nun:

$$
\beta=\frac{i \lambda}{4 a} \frac{\theta_{0}}{B_{0}} e^{-2 i k_{1} a} \text {. }
$$

$$
-\frac{G_{0}}{H_{0}}=r e^{i \psi},
$$

weiter folgt aus Gleichung (3), daB $\pi_{2}{ }^{2}={k_{2}}^{\prime \prime 2} \varrho^{\prime \prime 2}$ negativ imaginär, also $i \pi_{2}^{2}$ positiv reell ist; wir bezeichnen es mit $p^{2}$. Nach einer einfachen Rechnung erhält man dann für $q$ folgenden Wert:

$$
q=1+\frac{r p^{2}}{4} \frac{\lambda}{a} \cos \left(\frac{4 \pi}{\lambda} a-\psi\right)
$$

die Größen von der Ordnung $p^{4}$ sind hier, wie überall, vernachlässigt. 
Man sieht also, daß sich das Verhältnis zwischen dem beobachteten und dem theoretischen Werte periodisch ändert; die Periode ist gleich $\lambda / 2$. Die Lagen der Maxima bzw. Minima sind durch die Gleichung:

$$
\frac{4 \pi}{\lambda} a=2 n \pi+\psi
$$

bzw.

$$
\frac{4 \pi}{\lambda} a=(2 n+1) \pi+\psi
$$

bestimmt, sind also von den Konstanten des Thermoelementes unabhängig, da $\psi$ nur von der Beschaffenheit des vorderen Zylinders abhängt.

Ist der vordere Zylinder dielektrisch, so ist $\psi$ für $\lim \varrho^{\prime}=0$ gleich $\pi$, mit wachsendem $\varrho^{\prime}$ nimmt es ab. Aus den Gleichungen (42) und (42') folgt dann, daB sich die Maxima und Minima zu kleineren Werten von $a$ verschieben, wenn der Radius des vorderen Zylinders wächst. Ist dagegen der vordere Zylinder ein guter Leiter, so findet das Umgekehrte statt; für $\lim \varrho^{\prime}=0$ ist nämlich $\psi$ Null und mit wachsendem $\varrho^{\prime}$ nimmt es zu.

\begin{tabular}{|c|c|c|c|c|c|c|}
\hline \multirow[b]{2}{*}{$a$} & \multicolumn{3}{|c|}{$Q^{\prime}=0,8 \mathrm{~cm}$} & \multicolumn{3}{|c|}{$\rho^{\prime}=1,2 \mathrm{~cm}$} \\
\hline & $\begin{array}{l}\text { Theoret. } \\
\text { Wert der } \\
\text { mittleren } \\
\text { Feldint. }\end{array}$ & $\begin{array}{l}\text { Beob. } \\
\text { Wert der } \\
\text { mittleren } \\
\text { Feldint. }\end{array}$ & $q$ & $\begin{array}{l}\text { Theoret. } \\
\text { Wert der } \\
\text { mittleren } \\
\text { Feldint. }\end{array}$ & $\begin{array}{c}\text { Beob. } \\
\text { Wert der } \\
\text { mittleren } \\
\text { Feldint. }\end{array}$ & $q$ \\
\hline $4 \mathrm{~cm}$ & 1,848 & 2,414 & 1,306 & 0,273 & 0,371 & 1,357 \\
\hline 7 & 1,448 & 1,747 & 1,212 & 0,218 & 0,255 & 1,171 \\
\hline 10 & 1,282 & 1,414 & 1,103 & 0,227 & 0,218 & 0,959 \\
\hline 15 & 1,147 & 1,120 & 0,976 & 0,278 & 0,252 & 0,908 \\
\hline 20 & 1,076 & 1,003 & 0,932 & 0,327 & 0,309 & 0,945 \\
\hline 25 & 1,040 & 0,997 & 0,959 & 0,369 & 0,374 & 1,013 \\
\hline 30 & 1,017 & 1,027 & 1,010 & 0,405 & 0,426 & 1,051 \\
\hline 35 & 1,002 & 1,043 & 1,041 & 0,436 . & 0,452 & 1,036 \\
\hline 40 & 0,991 & 1,023 & 1,032 & 0,462 & 0,459 & 0,994 \\
\hline 50 & 0,979 & 0,953 & 0,974 & 0,505 & 0,492 & 0,973 \\
\hline 60 & 0,971 & 0,970 & 0,999 & 0,538 & 0,550 & 1,022 \\
\hline 70 & 0,963 & 0,983 & 1,020 & 0,564 & 0,566 & 1,003 \\
\hline 85 & 0,962 & 0,946 & 0,983 & 0,599 & 0,596 & 0,995 \\
\hline 100 & 0,961 & 0,976 & 1,015 & 0,626 & 0,630 & 1,007 \\
\hline
\end{tabular}

Tabelle II. 
In der vorstehenden Tab. II sind zwei Fälle berechnet worden: Wasserzylinder vom Radius $\varrho^{\prime}=0,8 \mathrm{~cm}$ bei der Wellenlänge $\lambda=62,8 \mathrm{~cm}$ und Wasserzylinder vom Radius $\varrho^{\prime}=1,2 \mathrm{~cm}$ bei derselben Wellenlänge. Das Thermoelement ist durch einen Platindraht $\left(\sigma=6,2 \cdot 10^{16}\right.$ elektrostat. Einh.) vom Radius $\varrho^{\prime \prime}=5 \cdot 10^{-4} \mathrm{~cm}$ ersetzt. Von den Messungen, die Grossmann ${ }^{1}$ ) durchgeführt hat, sind mit den hier berechneten Fällen am besten Wasserzylinder vom Radius $0,74 \mathrm{~cm}$ und $0,98 \mathrm{~cm}$ bei der Wellenlänge $\lambda=58 \mathrm{~cm}$ zu vergleichen. Die Überein. stimmung mit der Theorie ist ziemlich gut. Die Maxima und Minima liegen an gleichen Stellen; mit wachsendem Radius des Wasserzylinders zeigt sich eine deutliche Verschiebung des ersten Minimums zu kleineren Werten ron $a$, wie es die Theorie verlangt. Die Amplituden der periodischen Ände. rungen, so wie sie Grossmann beobachtet hat, sind viel größer als die hier berechneten; dies läBt sich bei der Konstruktion des Klemenčičschen Thermoelementes, das außerdem auf den Erreger abgestimmt war, erwarten.

Prag, 8. Februar 1913.

1) CI. Schaefer u. F. Grossmann, l. c. p. 497.

(Eingegangen 10. Februar 1913.) 